Tohoku Math. J.

65 (2013), 441-465

\title{
ON THE TWO-VARIABLES MAIN CONJECTURE FOR EXTENSIONS OF IMAGINARY QUADRATIC FIELDS
}

\author{
StéPhANe Viguié \\ (Received November 21, 2011, revised September 25, 2012)
}

\begin{abstract}
Let $p$ be a prime number at least 5, and let $k$ be an imaginary quadratic number field in which $p$ decomposes into two conjugate primes. Let $k_{\infty}$ be the unique $Z_{p^{-}}^{2}$ extension of $k$, and let $K_{\infty}$ be a finite extension of $k_{\infty}$, abelian over $k$. We prove that in $K_{\infty}$, the characteristic ideal of the projective limit of the $p$-class group coincides with the characteristic ideal of the projective limit of units modulo elliptic units. Our approach is based on Euler systems, which were first used in this context by Rubin.
\end{abstract}

1. Introduction. Let $p \notin\{2,3\}$ be a prime number, and let $k$ be an imaginary quadratic field in which $p$ decomposes into two distinct primes $\mathfrak{p}$ and $\overline{\mathfrak{p}}$. Let $k_{\infty}$ be the unique $\boldsymbol{Z}_{p}^{2}$-extension of $k$, and let $K_{\infty}$ be a finite extension of $k_{\infty}$, abelian over $k$. We set $G_{\infty}:=\operatorname{Gal}\left(K_{\infty} / k\right), G:=\operatorname{Gal}\left(K_{\infty} / k_{\infty}\right)$, and we fix a decomposition $G_{\infty}:=G \times \Gamma$, such that $\Gamma$ is a topological group isomorphic to $Z_{p}^{2}$.

For any global or local number field $L$, we denote by $\mathcal{O}_{L}$ the ring of integers of $L$, and for any ring $R$ we write $R^{\times}$the group of units in $R$. If $L$ is a finite abelian extension of $k$, let $\mathrm{A}_{L}$ be the $p$-part of the class group $\mathrm{Cl}\left(\mathcal{O}_{L}\right)$ of $\mathcal{O}_{L}$, and let $C_{L}$ be the group of elliptic units (see Definition 4.1). Then we set $\mathcal{E}_{L}:=\boldsymbol{Z}_{p} \otimes_{\boldsymbol{Z}} \mathcal{O}_{L}^{\times}$and $\mathcal{C}_{L}:=\boldsymbol{Z}_{p} \otimes_{\boldsymbol{Z}} C_{L}$. If $L$ is an infinite abelian extension of $k$, we define $\mathrm{A}_{L}, \mathcal{E}_{L}$ and $\mathcal{C}_{L}$ by taking projective limits over finite sub-extensions, under the norm maps. We set $\mathrm{A}_{\infty}:=\mathrm{A}_{K_{\infty}}, \mathcal{E}_{\infty}:=\mathcal{E}_{K_{\infty}}$, and $\mathcal{C}_{\infty}:=\mathcal{C}_{K_{\infty}}$.

For any profinite group $\mathcal{G}$, and any commutative ring $R$, we define the Iwasawa algebra $R[[\mathcal{G}]]:=\lim _{\leftarrow} R[\mathcal{H}]$, where the projective limit is taken over all finite quotient $\mathcal{H}$ of $\mathcal{G}$. We also set $\Lambda_{\mathcal{G}}:=Z_{p}[[\mathcal{G}]]$ and $\Lambda_{\infty}:=\Lambda_{G_{\infty}}$. Then $\mathrm{A}_{\infty}$ and $\mathcal{E}_{\infty} / \mathcal{C}_{\infty}$ are naturally $\Lambda_{\infty}$-modules. As we shall see below, they are finitely generated and torsion over $\Lambda_{\Gamma}$. For any finite extension $L / \boldsymbol{Q}_{p}$, it is well known that $\mathcal{O}_{L}[[\Gamma]]$ is a Noetherian local Krull ring. We also recall that $\mathcal{O}_{L}[[\Gamma]]$ is a unique factorization domain. Let $R$ be a Noetherian Krull ring which is not a field, and let $M$ be a finitely generated $R$-module. We say that $M$ is pseudo-null if and only if its localization at every height 1 prime ideal is zero. A morphism between two finitely generated $R$-modules is called pseudo-isomorphism if its kernel and its cokernel are pseudo-null. One may find height 1 prime ideals $\mathfrak{q}_{1}, \ldots, \mathfrak{q}_{r}$ in $R$, and nonnegative integers $n_{1}, \ldots, n_{r}$, such that there is a pseudo-isomorphism

$$
M \longrightarrow \bigoplus_{i=1}^{r} R / \mathfrak{q}_{i}^{n_{i}} .
$$

2010 Mathematics Subject Classification. Primary 11G16; Secondary 11R23, 11R65.

Key words and phrases. Elliptic units, Euler systems, Iwasawa theory. 
Moreover, the ideal $\mathfrak{q}_{1}^{n_{1}} \cdots \mathfrak{q}_{r}^{n_{r}}$ is uniquely determined by $M$, and is called the characteristic ideal of $M$, which we denote by $\operatorname{char}_{R}(M)$.

We denote by $\boldsymbol{C}_{p}$ the completion of an algebraic closure of $\boldsymbol{Q}_{p}$. Let $\chi: G \rightarrow \boldsymbol{C}_{p}^{\times}$be an irreducible character of $G$. Let $\boldsymbol{Q}_{p}(\chi) \subset \boldsymbol{C}_{p}$ be the extension of $\boldsymbol{Q}_{p}$ generated by the values of $\chi$. We denote by $\boldsymbol{Z}_{p}[\chi]$ the ring of integers of $\boldsymbol{Q}_{p}(\chi)$. The group $G$ acts on $\boldsymbol{Q}_{p}(\chi)$ through $\chi$. For any $\boldsymbol{Z}_{p}[G]$-module $Y$, we define its $\chi$-quotient by $Y_{\chi}:=\boldsymbol{Z}_{p}[\chi] \otimes \boldsymbol{Z}_{p}[G] Y$. Note that if $Y$ is a $\Lambda_{\infty}$-module, then $Y_{\chi}$ is a $Z_{p}[\chi][[\Gamma]]$-module in a natural way. As a particular case, $\Lambda_{\infty, \chi} \simeq Z_{p}[\chi][[\Gamma]]$.

The goal of the article is to prove Theorem 1.1 below, which is a formulation of the (two-variables) main conjecture. In [16, Theorem 4.1] and [17, Theorem 2], Rubin used Euler systems to prove the main conjectures for $\boldsymbol{Z}_{p^{-}}$or $\boldsymbol{Z}_{p}^{2}$-extensions of a finite abelian extension $F$ of $k$, where $p \nmid w_{k}[F: k], w_{k}$ being the number of roots of unity in $k$. More recently, Hassan Oukhaba adapted Rubin's method and obtained Theorem 1.1 for $p=2$, still under the condition $2 \nmid \# G$ (see [9]). Also, we draw the attention of the readers to a cohomological two-variables main conjecture, which has been recently proved by Johnson-Leung and Kings in $[8,5.2$ and 5.5]. In [8, 5.4], they recover the classical two-variables main conjecture in the case already treated by Rubin (i.e. $p \nmid \# G$ ). The contribution of the present paper is to extend Rubin's two-variables result to the general case, where we allow $p$ to divide \# $G$. One could try to deduce Theorem 1.1 below from the cohomological result of [8], but it is not clear that this strategy would succeed. Our approach is more elementary.

THEOREM 1.1. For all irreducible $\boldsymbol{C}_{p}$-character $\chi$ of $G$, the following equality holds:

$$
\operatorname{char}_{\Lambda_{\infty, \chi}}\left(\mathcal{E}_{\infty} / \mathcal{C}_{\infty}\right)_{\chi}=\operatorname{char}_{\Lambda_{\infty, \chi}} \mathrm{A}_{\infty, \chi} .
$$

Let us denote by $k_{\infty, 0}$ (resp. $k_{0, \infty}$ ) the unique $\boldsymbol{Z}_{p}$-extension of $k$ unramified outside of $\mathfrak{p}$ (resp. $\overline{\mathfrak{p}})$. We set $\Upsilon:=\operatorname{Gal}\left(K_{\infty} / k_{0, \infty} K\right)$ and $\bar{\Upsilon}:=\operatorname{Gal}\left(K_{\infty} / k_{\infty, 0} K\right)$, where $K$ is the subfield of $K_{\infty}$ fixed by $\Gamma$. For all $n \in N$, we set $\Upsilon_{n}:=\Upsilon^{p^{n}}$ and $\bar{\Upsilon}_{n}:=\bar{\Upsilon}^{p^{n}}$. Also we set $\Upsilon_{\infty}:=\{1\}$ and $\bar{\Upsilon}_{\infty}:=\{1\}$. For all $(n, \bar{n}) \in(N \cup\{\infty\})^{2}$, we denote by $K_{n, \bar{n}}$ the subfield of $K_{\infty}$ fixed by $\Gamma_{n, \bar{n}}:=\Upsilon_{n} \bar{\Upsilon}_{\bar{n}}$. We choose topological generators $\gamma$ and $\bar{\gamma}$ of $\Upsilon$ and $\bar{\Upsilon}$, respectively, and for all $n \in N$ we set $\gamma_{n}:=\gamma^{p^{n}}$ and $\bar{\gamma}_{n}:=\bar{\gamma} p^{n}$.

We fix an irreducible $\boldsymbol{C}_{p}$-character $\chi$ of $G$, and we set $\psi: G \rightarrow \boldsymbol{Q}_{p}, \sigma \mapsto \operatorname{Tr}(\chi(\sigma))$, where $\operatorname{Tr}$ is the trace map for the extension $\boldsymbol{Q}_{p}(\chi) / \boldsymbol{Q}_{p}$. We denote by $\boldsymbol{e}_{\chi}$ (resp. $\boldsymbol{e}_{\psi}$ ) the idempotent of $\boldsymbol{Q}_{p}(\chi)[G]$ (resp. $\boldsymbol{Q}_{p}[G]$ ) attached to $\chi($ resp. $\psi$ ), i.e.,

$$
e_{\chi}:=(\# G)^{-1} \sum_{g \in G} \chi(g) g^{-1} \text { and } e_{\psi}:=(\# G)^{-1} \sum_{g \in G} \psi(g) g^{-1} .
$$

For any closed subgroup $H$ of $G_{\infty}$, and any $\Lambda_{\infty}$-module $N$, we denote by $N^{H}$ the module of $H$-invariants of $N$, and we denote by $N_{H}$ the $\Lambda_{\infty}$-module of coinvariants of $N$. Let $\mathcal{F}$ be the set of all finite extensions $F \subset K_{\infty}$ of $k$. Let $\left(M_{F}\right)_{F \in \mathcal{F}}$ be a projective system of $\Lambda_{\infty}$-modules. We assume that for all $F \in \mathcal{F}, M_{F}$ is invariant under $\operatorname{Gal}\left(K_{\infty} / F\right)$. For all $F \subset F^{\prime}$ in $\mathcal{F}$, let $\mathrm{N}_{F^{\prime} / F}: M_{F^{\prime}} \rightarrow M_{F}$ be the transition map (in all the case encountered in the sequel, these transition maps are the usual norm maps). For any extension $L \subseteq K_{\infty}$ of $k$, 
we set $M_{L}:=\lim _{F} M_{F}$, where the projective limit is taken over all $F \in \mathcal{F}$ such that $F \subset L$. We denote by $\pi_{L}^{M}:\left(M_{K_{\infty}}\right)_{\Gamma_{L}} \rightarrow M_{L}$ and $\pi_{L}^{M_{\chi}}:\left(M_{K_{\infty}, \chi}\right)_{\Gamma_{L}} \rightarrow M_{L, \chi}$ the canonical maps, where $\Gamma_{L}:=\operatorname{Gal}\left(K_{\infty} / L\right)$. Notice that $M_{K_{\infty}, \chi}$ may not be equal to $\lim _{F} M_{F, \chi}$. For any two extensions $L \subset L^{\prime} \subseteq K_{\infty}$ of $k$, we denote by $\mathrm{N}_{L^{\prime} / L}: M_{L^{\prime}} \rightarrow M_{L}$ the canonical map obtained from the transition maps by taking the projective limit. For all $(n, \bar{n}) \in(N \cup\{\infty\})^{2}, M_{K_{n, \bar{n}}}$, $\pi_{K_{n, \bar{n}}}^{M}$ and $\pi_{K_{n, \bar{n}}}^{M_{\chi}}$ are respectively denoted by $M_{n, \bar{n}}, \pi_{n, \bar{n}}^{M}$ and $\pi_{n, \bar{n}}^{M_{\chi}}$. We set $M_{\infty}:=M_{K_{\infty}}$.

2. Semilocal units and ideal class group. Let $F$ be an abelian extension of $k$. If $[F: k]<\infty$, for any maximal ideal $\mathfrak{r}$ of $\mathcal{O}_{F}$, we denote by $F_{\mathfrak{r}}$ the completion of $F$ at $\mathfrak{r}$. Then we write $\mathcal{U}_{F}$ for the pro- $p$-completion of $\prod_{\mathfrak{q} \mid \mathfrak{p}} \mathcal{O}_{F_{\mathfrak{q}}}^{\times}$, where the product is over all primes of $F$ above $\mathfrak{p}$. If $[F: k]$ is not finite, $\mathcal{U}_{F}$ is defined in accordance with our conventions (Section 1 ). For any field $L$ and any $n \in N$, we denote by $\mu_{n}(L)$ the group of $n$-th roots of unity. We set $\mu(L):=\bigcup_{n>0} \mu_{n}(L)$ and $\mu_{p^{\infty}}(L):=\bigcup_{n \in N} \mu_{p^{n}}(L)$. We denote by $D$ and $\bar{D}$ the respective decomposition groups of $\mathfrak{p}$ and $\overline{\mathfrak{p}}$ in $K_{\infty} / k$.

THEOREM 2.1. Let $\mathcal{U}_{\infty}:=\mathcal{U}_{K_{\infty}}$. For any prime $\mathfrak{q}$ of $K_{\infty}$ above $\mathfrak{p}$, we set $K_{\infty, \mathfrak{q}}:=$ $\bigcup_{(n, \bar{n}) \in N^{2}} K_{n, \bar{n}, \mathfrak{q}}$, where $K_{n, \bar{n}, \mathfrak{q}}$ is the completion of $K_{n, \bar{n}}$ at $\mathfrak{q}$. Then there is an exact sequence

$$
0 \longrightarrow \mathcal{U}_{\infty} \longrightarrow \Lambda_{\infty} \longrightarrow \prod_{\mathfrak{q} \mid \mathfrak{p}} T\left(\mu_{p^{\infty}}\left(K_{\infty, \mathfrak{q}}\right)\right) \longrightarrow 0
$$

where the product is over all prime $\mathfrak{q}$ of $K_{\infty}$ above $\mathfrak{p}$, and where the letter $T$ stands for the Tate module.

Proof. By class field theory and since $k_{\mathfrak{p}} \simeq \boldsymbol{Q}_{p}, D$ is a quotient of $(\boldsymbol{Z} /(p-1) \boldsymbol{Z}) \times$ $\widehat{\boldsymbol{Z}} \times \boldsymbol{Z}_{p}$, where $\widehat{\boldsymbol{Z}}$ is the profinite completion of $\boldsymbol{Z}$. Moreover, the $\boldsymbol{Z}_{p}$-rank of the pro- $p$-part of $D$ is 2 . From these two facts we deduce that $D \simeq \Delta \times Z_{p}^{2}$ for some finite group $\Delta$ of order prime to $p$. Then we can apply [21, Théorème].

LEMMA 2.2. Let $F \subsetneq K_{\infty}$ be an extension of $K$, such that $K_{\infty} / F$ is unramified at all the places above $\mathfrak{p}$. Then $\pi_{F}^{\mathcal{U}}:\left(\mathcal{U}_{\infty}\right)_{\Gamma_{F}} \rightarrow \mathcal{U}_{F}$ is an isomorphism, where $\Gamma_{F}:=\operatorname{Gal}\left(K_{\infty} / F\right)$.

PROOF. Let $\tilde{F}$ be the subfield of $K_{\infty}$ fixed by $\Gamma_{F} \cap D$. Since all the primes of $F$ above $\mathfrak{p}$ are totally split in $\tilde{F} / F$, we have $\left(\mathcal{U}_{\tilde{F}}\right)_{\Gamma_{F}} \simeq \mathcal{U}_{F}$. Thus we are reduced to prove Lemma 2.2 when $F=\tilde{F}$. In that case we have $\operatorname{Ker}\left(\pi_{F}^{\mathcal{U}}\right)=0$ by [21, Lemme 5.2 (i)], and $\pi_{F}^{\mathcal{U}}$ is surjective by local class field theory, since $K_{\infty} / F$ is unramified at all the places above $\mathfrak{p}$.

Let $\Upsilon^{\prime}$ be a subgroup of $\Gamma$ such that $\Gamma=\bar{\Upsilon} \times \Upsilon^{\prime}$, and let $\gamma^{\prime}$ be a topological generator of $\Upsilon^{\prime}$. For all $n \in N$, we set $\gamma_{n}^{\prime}:=\left(\gamma^{\prime}\right)^{p^{n}}$.

Lemma 2.3. Let $\delta^{\prime} \in N$ be such that $\Upsilon^{\prime} \cap D=\Upsilon_{\delta^{\prime}}^{\prime}$. For all $(n, \bar{n}) \in N^{2}, \gamma_{\delta^{\prime}}^{\prime}-1$ annihilates the kernel and the cokernel of the natural map $\left(\mathcal{U}_{\infty, \bar{n}}\right)_{n}^{\prime} \rightarrow \mathcal{U}_{K_{n, \bar{n}}^{\prime}}$, where $K_{n, \bar{n}}^{\prime}$ is the subfield of $K_{\infty}$ fixed by $\bar{\Upsilon}_{\bar{n}} \Upsilon_{n}^{\prime}$.

Proof. Lemma 2.3 is obvious if $n \leq \delta^{\prime}$. We fix $\bar{n} \in N$ and $n>\delta^{\prime}$. Let $\mathfrak{P}$ be a maximal ideal of $\mathcal{O}_{K_{\infty}}$ such that $\mathfrak{P} \cap \mathcal{O}_{k}=\mathfrak{p}$. For all $m \in N$, we denote by $\mathcal{U}_{m}^{\prime}$ the pro- $p$-completion 
of $\mathcal{O}_{K_{m, \bar{n}, \mathfrak{P}}^{\prime}}^{\times}$and we set $\mathcal{U}_{\infty}^{\prime}:=\lim _{\leftarrow \geq \delta^{\prime}} \mathcal{U}_{m}^{\prime}$, projective limit under the norm maps. Then we are reduced to show that $\gamma_{\delta^{\prime}}^{\prime}-1$ annihilates the kernel and the cokernel of the natural map $\pi_{n}^{\prime}:\left(\mathcal{U}_{\infty}^{\prime}\right)_{\Upsilon_{n}^{\prime}} \rightarrow \mathcal{U}_{n}^{\prime}$. For all $m \in N \cup\{\infty\}$, we denote by $M_{m}$ the maximal abelian pro$p$-extension of $K_{m, \bar{n}, \mathfrak{P}}^{\prime}$, and $L_{m}$ the maximal unramified abelian pro- $p$-extension of $K_{m, \bar{n}, \mathfrak{P}}^{\prime}$. Remark that $L_{\infty}$ is abelian over $k_{\mathfrak{p}}$. For all $m \geq \delta^{\prime}$, local class field theory gives us an isomorphism $\phi_{m}: \mathcal{U}_{m}^{\prime} \rightarrow \operatorname{Gal}\left(M_{m} / L_{m}\right)$. Taking projective limits under the norm and the restriction maps, we obtain an isomorphism $\phi_{\infty}: \mathcal{U}_{\infty}^{\prime} \rightarrow \operatorname{Gal}\left(M_{\infty} / L_{\infty}\right)$ of $\Lambda_{D}$-modules, which induces an isomorphism

$$
\operatorname{Cok}\left(\pi_{n}^{\prime}\right) \simeq \operatorname{Gal}\left(L_{\infty} / L_{n}\right)
$$

The restriction map $\operatorname{Gal}\left(M_{\infty} / K_{\infty, \bar{n}, \mathfrak{P}}\right) \rightarrow \operatorname{Gal}\left(M_{n} / K_{n, \bar{n}, \mathfrak{P}}^{\prime}\right)$ induces an isomorphism

$$
\operatorname{Gal}\left(M_{\infty} / K_{\infty, \bar{n}, \mathfrak{P}}\right)_{\Upsilon_{n}^{\prime}} \simeq \operatorname{Gal}\left(M_{n} / K_{\infty, \bar{n}, \mathfrak{P}}\right)
$$

Since $\mu_{p^{\infty}}\left(K_{\infty, \bar{n}, \mathfrak{P}}\right)$ is finite by class field theory, we deduce from [6, Theorem 25 (i)] that

$$
\operatorname{Gal}\left(M_{\infty} / K_{\infty, \bar{n}, \mathfrak{P}}\right)^{\Upsilon_{n}^{\prime}}=0
$$

Since $L_{\infty}$ is abelian over $k_{\mathfrak{p}}$, we have

$$
\operatorname{Gal}\left(L_{\infty} / K_{\infty, \bar{n}, \mathfrak{P}}\right)^{\Upsilon_{n}^{\prime}}=\operatorname{Gal}\left(L_{\infty} / K_{\infty, \bar{n}, \mathfrak{P}}\right) \simeq \operatorname{Gal}\left(L_{\infty} / K_{\infty, \bar{n}, \mathfrak{P}}\right)_{n}^{\prime}
$$

The exact sequence $0 \rightarrow \mathcal{U}_{\infty}^{\prime} \rightarrow \operatorname{Gal}\left(M_{\infty} / K_{\infty, \bar{n}, \mathfrak{P}}\right) \rightarrow \operatorname{Gal}\left(L_{\infty} / K_{\infty, \bar{n}, \mathfrak{P}}\right)$, together with (2.3), (2.4) and (2.5), gives us the following commutative diagram with exact rows:

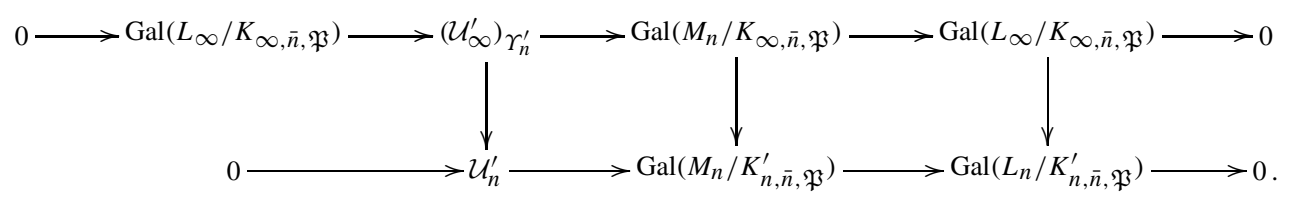

From this diagram we deduce an isomorphism of $\Lambda_{D}$-modules $\operatorname{Ker}\left(\pi_{n}^{\prime}\right) \simeq \operatorname{Gal}\left(L_{\infty} / K_{\infty, \bar{n}, \mathfrak{P}}\right)$. Since $L_{\infty}$ is abelian over $k_{\mathfrak{p}}$, Lemma 2.3 follows from this last isomorphism and (2.2).

For any abelian extension $F$ of $k$, we denote by $\mathrm{M}_{\mathfrak{p}}(F)$ the maximal pro- $p$-extension of $F$ which is unramified outside the primes above $\mathfrak{p}$. We set $\mathrm{B}_{F}:=\operatorname{Gal}\left(\mathrm{M}_{\mathfrak{p}}(F) / F\right)$. For any closed subgroup $H$ of $G_{\infty}$, we denote by $\mathcal{J}_{H}$ the ideal of $\Lambda_{\infty}$ generated by all the $h-1$, with $h \in H$. We set $\mathcal{J}_{\infty}:=\mathcal{J}_{G_{\infty}}$.

THEOREM 2.4. Let $F \subsetneq K_{\infty}$ be an extension of $K$. Then we have the following.

(i) $\mathrm{B}_{F}$ is finitely generated over $\Lambda_{\mathrm{Gal}(F / K)} . \mathrm{B}_{\infty}:=\mathrm{B}_{K_{\infty}}$ is finitely generated and torsion over $\Lambda_{\infty}$.

(ii) $\operatorname{Ker}\left(\pi_{F}^{\mathrm{B}}\right)$ is finitely generated over $\boldsymbol{Z}_{p}$, and if $\operatorname{Gal}\left(K_{\infty} / F\right) \subseteq \bar{\Upsilon}$ then it is annihilated by $\mathcal{J}_{\bar{D}}$.

(iii) $\operatorname{Cok}\left(\pi_{F}^{\mathrm{B}}\right)$ is finitely generated over $\boldsymbol{Z}_{p}$, annihilated by $\mathcal{J}_{\infty}$, and if $\operatorname{Gal}\left(K_{\infty} / F\right) \subseteq$ $\bar{\Upsilon}$ then it is finite.

(iv) If $\operatorname{Gal}\left(K_{\infty} / F\right) \subseteq \bar{\Upsilon}$ then we have the exact sequence 


$$
0 \longrightarrow \operatorname{Ker}\left(\pi_{F}^{\mathrm{B}}\right) \longrightarrow \Lambda_{F} / \mathcal{J}_{\bar{D}} \Lambda_{F} \longrightarrow Z_{p} \longrightarrow \operatorname{Cok}\left(\pi_{F}^{\mathrm{B}}\right) \longrightarrow 0
$$

where $\Lambda_{F}:=\Lambda_{\mathrm{Gal}(F / K)}$.

PROOF. We refer the readers to [16, Theorem 5.3].

PROPOSITION 2.5. The module $\mathrm{B}_{\infty}$ enjoys the following properties.

(i) $\mathrm{B}_{\infty}$ has no nonzero pseudo-null $\Lambda_{\Gamma}$-submodule.

(ii) $\mathrm{B}_{\infty}$ has no torsion over $\boldsymbol{Z}_{p}$.

(iii) For all $n \in N,\left(\mathrm{~B}_{\infty}\right)^{\bar{\Upsilon}_{n}}=0$.

PROOF. (i) is proved in [11, Théorème 23]. We identify $\mathrm{B}_{\infty}$ with $\lim _{n} \mathrm{~B}_{\infty, n}$. Let $m \in$ $N$ and $x:=\left(x_{n}\right)_{n \in N}$ be an element of $\mathrm{B}_{\infty}$ such that $p^{m} x=0$. For all $\overleftarrow{n}_{n}^{n} \in N, \Lambda_{\Upsilon^{\prime}} x_{n}$ is a submodule of $\mathrm{B}_{\infty, n}$, and a quotient of $\Lambda_{\Upsilon^{\prime}} /\left(p^{m}\right)$. By the result of Gillard [3, 3.4. Théorème], the Iwasawa $\mu$-invariant $\mu_{\Lambda_{\Upsilon^{\prime}}}\left(\mathrm{B}_{\infty, n}\right)$ is 0 . Hence $\Lambda_{\Upsilon^{\prime}} x_{n}$ must be pseudo-null over $\Lambda_{\Upsilon^{\prime}}$. By the result of Greenberg [4, end of Section 4], $\mathrm{B}_{\infty, n}$ has no nonzero finite $\Lambda_{\Upsilon^{\prime}}$-submodule. Then $x_{n}=0$, for all $n \in N$, and (ii) is verified. Let us fix $n \in N$. From Theorem 2.4 and the exact sequence

$$
0 \longrightarrow \operatorname{Ker}\left(\pi_{\infty, n}^{\mathrm{B}}\right) \longrightarrow\left(\mathrm{B}_{\infty}\right)_{\bar{\Upsilon}_{n}} \longrightarrow \mathrm{B}_{\infty, n} \longrightarrow \operatorname{Cok}\left(\pi_{\infty, n}^{\mathrm{B}}\right) \longrightarrow 0
$$

we deduce that $\left(\mathrm{B}_{\infty}\right) \bar{\Upsilon}_{n}$ is torsion over $\Lambda_{\Upsilon^{\prime}}$. Then by [11, Lemme 4 (2)], $\left(\mathrm{B}_{\infty}\right)^{\bar{\Upsilon}_{n}}$ is torsion over $\Lambda_{\Upsilon^{\prime}}$. By (ii) we deduce that $\left(\mathrm{B}_{\infty}\right)^{\bar{\Upsilon}_{n}}$ is finitely generated over $\boldsymbol{Z}_{p}$. Then $\left(\mathrm{B}_{\infty}\right)^{\bar{\Upsilon}_{n}}$ is pseudo-null over $\Lambda_{\Gamma}$, and (i) implies (iii).

For any abelian extension $F$ of $k$, we denote by $\mathrm{M}(F)$ the maximal unramified pro- $p$ extension of $F$. We set $\mathrm{A}_{F}:=\operatorname{Gal}(\mathrm{M}(F) / F)$. Hence if $[F: k]$ is finite, $\mathrm{A}_{F}$ is identified to the $p$-part of $\mathrm{Cl}\left(\mathcal{O}_{F}\right)$ by class field theory.

THEOREM 2.6. Let $F \subsetneq K_{\infty}$ be an extension of $K$. Then we have the following. $\Lambda_{\infty}$

(i) $\mathrm{A}_{F}$ is finitely generated over $\Lambda_{\mathrm{Gal}(F / K)} . \mathrm{A}_{\infty}$ is finitely generated and torsion over

(ii) $\operatorname{Ker}\left(\pi_{F}^{\mathrm{A}}\right)$ is finitely generated over $\boldsymbol{Z}_{p}$, and annihilated by $\mathcal{J}_{D} \mathcal{J}_{\bar{D}} \mathcal{J}_{\infty}$.

(iii) $\operatorname{Cok}\left(\pi_{F}^{\mathrm{A}}\right)$ is finitely generated over $\boldsymbol{Z}_{p}$, annihilated by $\mathcal{J}_{\infty}$, and if $[F: K]<\infty$ then it is finite.

Proof. We refer the readers to [16, Theorem 5.4].

REMARK 2.7. Proceeding as in the proof of Proposition 2.5, one can show that for any closed subgroup $H$ of $\Gamma,\left(\mathrm{A}_{\infty}\right)_{H}$ and $\left(\mathrm{A}_{\infty}\right)^{H}$ are finitely generated torsion $\Lambda_{\Gamma / H}$-modules. As a consequence, for all $g \in \Gamma \backslash\{1\}$, $\operatorname{char}_{\Lambda_{\Gamma}}\left(\mathrm{A}_{\infty}\right)$ is prime to $1-g$.

Proposition 2.8. We have $\mu_{\Lambda_{\gamma^{\prime}}}\left(\mathrm{A}_{\infty}\right) \bar{\gamma}=0$. As a consequence, the image of $\operatorname{char}_{\Lambda_{\infty, \chi}}\left(\mathrm{A}_{\infty, \chi}\right)$ in $\boldsymbol{Z}_{p}[\chi]\left[\left[\Upsilon^{\prime}\right]\right]$ is prime to $\mathfrak{u}_{\chi}$, where $\mathfrak{u}_{\chi}$ is a uniformizer of $\boldsymbol{Z}_{p}[\chi]$. 
Proof. We have $\mu_{\Lambda_{\gamma^{\prime}}}\left(\operatorname{Ker}\left(\pi_{\infty, 0}^{\mathrm{A}}\right)\right)=0$ because $\operatorname{Ker}\left(\pi_{\infty, 0}^{\mathrm{A}}\right)$ is finitely generated over $Z_{p}$. But $\mu_{\Lambda_{\gamma^{\prime}}}\left(\mathrm{A}_{\infty, 0}\right)=0$ by Gillard's result [3, 3.4. Théorème], and then we deduce $\mu_{\Lambda_{\Upsilon^{\prime}}}\left(\mathrm{A}_{\infty}\right) \bar{\gamma}=0$. The rest of Proposition 2.8 follows by [11, Lemme 4 (2)].

LemMA 2.9. Let $(n, \bar{n}) \in N^{2}$. Then $\mathcal{J}_{D} \mathcal{J}_{\bar{D}} \mathcal{J}_{\infty}^{2} \operatorname{Ker}\left(\pi_{n, \bar{n}}^{\mathrm{A}_{\bar{\chi}}}\right)=0, \operatorname{Cok}\left(\pi_{n, \bar{n}}^{\mathrm{A}_{\chi}}\right)$ is finite and annihilated by $\mathcal{J}_{\infty}$.

PROOF. The assertions about $\operatorname{Cok}\left(\pi_{n, \bar{n}}^{\mathrm{A}_{\chi}}\right)$ follow from Theorem 2.6 (iii). We have the obvious exact sequences

$$
\operatorname{Ker}\left(\pi_{n, \bar{n}}^{\mathrm{A}}\right)_{\chi} \longrightarrow\left(\mathrm{A}_{\infty, \chi}\right)_{\Gamma_{n, \bar{n}}} \longrightarrow \operatorname{Im}\left(\pi_{n, \bar{n}}^{\mathrm{A}}\right)_{\chi} \longrightarrow 0
$$

$$
\operatorname{Tor}_{\Lambda_{\infty}}^{1}\left(\operatorname{Cok}\left(\pi_{n, \bar{n}}^{\mathrm{A}}\right), \Lambda_{\infty, \chi}\right) \longrightarrow \operatorname{Im}\left(\pi_{n, \bar{n}}^{\mathrm{A}}\right)_{\chi} \longrightarrow \mathrm{A}_{n, \bar{n}, \chi} \longrightarrow \operatorname{Cok}\left(\pi_{n, \bar{n}}^{\mathrm{A}}\right)_{\chi} \longrightarrow 0 .
$$

By Theorem 2.6 we have $\mathcal{J}_{\infty} \operatorname{Tor}_{\Lambda_{\infty}}^{1}\left(\operatorname{Cok}\left(\pi_{n, \bar{n}}^{\mathrm{A}}\right), \Lambda_{\infty, \chi}\right)=0$ and $\mathcal{J}_{D} \mathcal{J}_{\bar{D}} \mathcal{J}_{\infty} \operatorname{Ker}\left(\pi_{n, \bar{n}}^{\mathrm{A}}\right)_{\chi}=0$, from which we derive the lemma.

Let us fix a pseudo-isomorphism $\tau_{\infty, \chi}: \mathrm{A}_{\infty, \chi} \rightarrow \bigoplus_{j=1}^{s}\left(\Lambda_{\infty, \chi} /\left(P_{j}\right)\right)$, where $P_{1}, \ldots$, $P_{S}$ are nonzero elements of $\Lambda_{\infty, \chi}$.

LEMMA 2.10. There is an ideal $\mathcal{I}_{0}$ of $\Lambda_{\infty, \chi}$ of height greater than 1 , and for all $(n, \bar{n}) \in N^{2}$ there is a morphism

$$
\tau_{n, \bar{n}, \chi}: \mathrm{A}_{n, \bar{n}, \chi} \longrightarrow \bigoplus_{j=1}^{s}\left(\Lambda_{\infty, \chi} /\left(P_{j}, \gamma_{n}-1, \bar{\gamma}_{\bar{n}}-1\right)\right)
$$

such that $\operatorname{Cok}\left(\tau_{n, \bar{n}, \chi}\right)$ is annihilated by $\mathcal{I}_{0}\left(\gamma_{\delta^{\prime}}^{\prime}-1\right)^{2}\left(\bar{\gamma}_{\bar{\delta}}-1\right)^{2}(\bar{\gamma}-1)$, where $\bar{\delta} \in N$ is such that $\bar{\Upsilon}_{\bar{\delta}}=\bar{D} \cap \bar{\Upsilon}$.

PROOF. For all $x \in \mathrm{A}_{n, \bar{n}, \chi}$ we choose $\tilde{x} \in\left(\mathrm{A}_{\infty, \chi}\right)_{\Gamma_{n, \bar{n}}}$ such that $\pi_{n, \bar{n}}^{\mathrm{A}_{\chi}}(\tilde{x})=(\bar{\gamma}-1) x$. Such a choice is possible by Lemma 2.9. Then we set $\tau_{n, \bar{n}, \chi}(x):=\left(\gamma_{\delta^{\prime}}^{\prime}-1\right)^{2}\left(\bar{\gamma}_{\bar{\delta}}-1\right)^{2} \tilde{\tau}_{n, \bar{n}, \chi}(\tilde{x})$, where

$$
\tilde{\tau}_{n, \bar{n}, \chi}:\left(\mathrm{A}_{\infty, \chi}\right)_{\Gamma_{n, \bar{n}}} \longrightarrow \bigoplus_{j=1}^{s}\left(\Lambda_{\infty, \chi} /\left(P_{j}, \gamma_{n}-1, \bar{\gamma}_{\bar{n}}-1\right)\right)
$$

is obtained from $\tau_{\infty, \chi}$ by taking the quotients. It follows from Lemma 2.9 that $\tau_{n, \bar{n}, \chi}$ is well defined. Lemma 2.10 is satisfied if we let $\mathcal{I}_{0}$ be the annihilator of $\operatorname{Cok}\left(\tau_{\infty, \chi}\right)$ over $\Lambda_{\infty, \chi}$.

3. Global units. Let $F$ be an abelian extension of $k$. If $[F: k]<\infty$, we set $\mathcal{E}_{F}:=$ $\boldsymbol{Z}_{p} \otimes_{\boldsymbol{Z}} \mathcal{O}_{F}^{\times}$. If $[F: k]=\infty$, then we set $\mathcal{E}_{F}:=\lim _{\leftarrow} \mathcal{E}_{L}$, where the limit is taken over all finite subextensions $L \subset F$ of $k$ under the norm maps. $\overleftarrow{\text { In }}$ all cases, by an appropriate version of the Leopoldt conjecture which is known to be true for abelian extensions of imaginary quadratic fields, one can identify $\mathcal{E}_{F}$ with a submodule of $\mathcal{U}_{F}$. We set $\mathcal{E}_{\infty}:=\mathcal{E}_{K_{\infty}}$. 
LEMMA 3.1. For any $n \in N$, we have the following commutative diagram with exact rows:

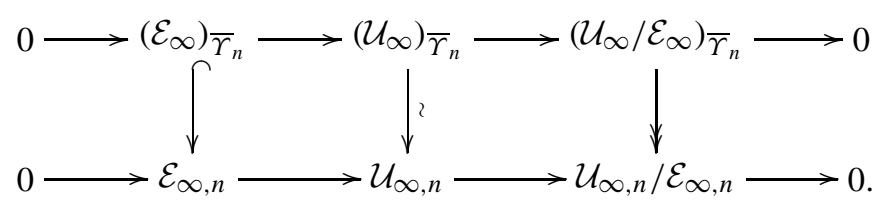

Proof. By class field theory, for any abelian extension $F$ of $k$ we have an exact sequence

$$
0 \longrightarrow \mathcal{E}_{F} \longrightarrow \mathcal{U}_{F} \longrightarrow \mathrm{B}_{F} \longrightarrow \mathrm{A}_{F} \longrightarrow 0
$$

By Proposition 2.5 (iii) and (3.2) applied to $F=K_{\infty}$, we have $\left(\mathcal{U}_{\infty} / \mathcal{E}_{\infty}\right)^{\bar{\Upsilon}_{n}}=0$. Moreover $\left(\mathcal{U}_{\infty}\right)_{\bar{\Upsilon}_{n}} \simeq \mathcal{U}_{\infty, n}$ by Lemma 2.2. Then we deduce Lemma 3.1 from the tautological exact sequence $0 \longrightarrow \mathcal{E}_{\infty} \longrightarrow \mathcal{U}_{\infty} \longrightarrow \mathcal{U}_{\infty} / \mathcal{E}_{\infty} \longrightarrow 0$.

LEMMA 3.2. For any $\bar{n} \in N$, there is $c_{\bar{n}} \in N$ such that for all integers $n \geq \bar{n}$,

$p^{c_{\bar{n}}}\left(\gamma_{\delta^{\prime}}^{\prime}-1\right)$ annihilates $\operatorname{Ker}\left(\left(\mathcal{E}_{\infty, \bar{n}}\right)_{\Upsilon_{n}} \rightarrow \mathcal{E}_{n, \bar{n}}\right)$ and $\operatorname{Cok}\left(\left(\mathcal{E}_{\infty, \bar{n}}\right)_{\Upsilon_{n}} \rightarrow \mathcal{E}_{n, \bar{n}}\right)$.

Proof. Let us fix $\bar{n} \in N$. For all $n \in N$, we recall that $K_{n, \bar{n}}^{\prime}$ is the subfield of $K_{\infty}$ fixed by $\Upsilon_{n}^{\prime} \bar{\Upsilon}_{\bar{n}}$. We also set $K_{\infty, \bar{n}}^{\prime}:=K_{\infty, \bar{n}}$. From (3.2) applied to $K_{\infty, \bar{n}}$ and $K_{n, \bar{n}}^{\prime}$, we deduce the commutative diagram with exact rows below:

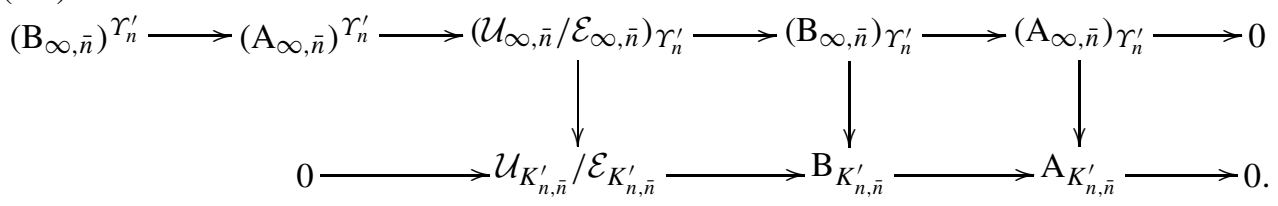

Since $\left(\mathrm{B}_{\infty, \bar{n}}\right)_{\Upsilon_{n}^{\prime}}=\operatorname{Gal}\left(\mathrm{M}_{\mathfrak{p}}\left(K_{n, \bar{n}}^{\prime}\right) / K_{\infty, \bar{n}}\right)$, the map $\left(\mathrm{B}_{\infty, \bar{n}}\right)_{\Upsilon_{n}^{\prime}} \rightarrow \mathrm{B}_{K_{n, \bar{n}}^{\prime}}$ is injective. Moreover, it is well known that $\left(\mathrm{B}_{\infty, \bar{n}}\right)^{\Upsilon_{n}^{\prime}}=0$ (see [16, Theorem $\left.5.3(\mathrm{v})\right]$ ), hence from (3.3) we deduce that $\operatorname{Ker}\left(\left(\mathcal{U}_{\infty, \bar{n}} / \mathcal{E}_{\infty, \bar{n}}\right)_{\Upsilon_{n}^{\prime}} \rightarrow \mathcal{U}_{K_{n, \bar{n}}^{\prime}} / \mathcal{E}_{K_{n, \bar{n}}^{\prime}}\right) \simeq\left(\mathrm{A}_{\infty, \bar{n}}\right)^{\Upsilon_{n}^{\prime}}$. Since $\mathrm{A}_{\infty, \bar{n}}$ is Noetherian and since $\left(\mathrm{A}_{\infty, \bar{n}}\right)^{\Upsilon_{n}^{\prime}}$ is finite (see [15, Theorem 1.4]), we can find $c_{\bar{n}} \in \boldsymbol{N}$ such that for all $m \in N$,

$$
p^{c_{\bar{n}}} \quad \text { annihilates } \operatorname{Ker}\left(\left(\mathcal{U}_{\infty, \bar{n}} / \mathcal{E}_{\infty, \bar{n}}\right)_{\Upsilon_{m}^{\prime}} \rightarrow \mathcal{U}_{K_{m, \bar{n}}^{\prime}} / \mathcal{E}_{K_{m, \bar{n}}^{\prime}}\right) .
$$

From (3.2) we have $\left(\mathcal{U}_{\infty, \bar{n}} / \mathcal{E}_{\infty, \bar{n}}\right)^{\Upsilon_{m}^{\prime} \hookrightarrow}\left(\mathrm{B}_{\infty, \bar{n}}\right)^{\Upsilon_{m}^{\prime}}=0$, and the commutative diagram with exact rows below:

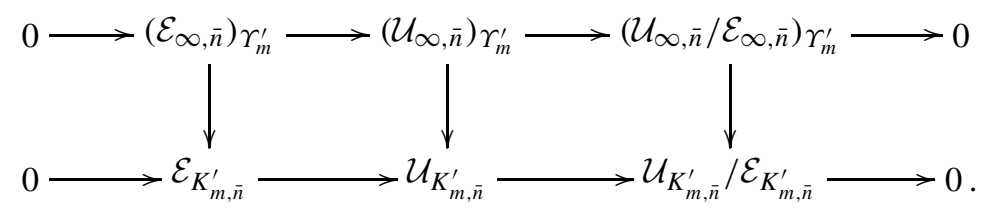


Applying the snake lemma for (3.5), we see that $\operatorname{Ker}\left(\left(\mathcal{E}_{\infty, \bar{n}}\right)_{\Upsilon_{m}^{\prime}} \rightarrow \mathcal{E}_{K_{m, \bar{n}}^{\prime}}\right)$ is a submodule of $\operatorname{Ker}\left(\left(\mathcal{U}_{\infty, \bar{n}}\right)_{\Upsilon_{m}^{\prime}} \rightarrow \mathcal{U}_{K_{m, \bar{n}}^{\prime}}\right)$, which is annihilated by $\left(\gamma_{\delta^{\prime}}^{\prime}-1\right)$ from Lemma 2.3. Moreover, the snake lemma gives an exact sequence

$$
0 \longrightarrow Q \longrightarrow \operatorname{Cok}\left(\left(\mathcal{E}_{\infty, \bar{n}}\right)_{\Upsilon_{m}^{\prime}} \rightarrow \mathcal{E}_{K_{m, \bar{n}}^{\prime}}\right) \longrightarrow S \longrightarrow 0,
$$

where $Q$ is a quotient of $\operatorname{Ker}\left(\left(\mathcal{U}_{\infty, \bar{n}} / \mathcal{E}_{\infty, \bar{n}}\right)_{\Upsilon_{m}^{\prime}} \rightarrow \mathcal{U}_{K_{m, \bar{n}}^{\prime}} / \mathcal{E}_{K_{m, \bar{n}}^{\prime}}\right)$ and $S$ is a submodule of $\operatorname{Cok}\left(\left(\mathcal{U}_{\infty, \bar{n}}\right)_{\Upsilon_{m}^{\prime}} \rightarrow \mathcal{U}_{K_{m, \bar{n}}^{\prime}}\right)$. Then by (3.4) and Lemma 2.3, $p^{c_{\bar{n}}}\left(\gamma_{\delta^{\prime}}^{\prime}-1\right)$ annihilates $\operatorname{Cok}\left(\left(\mathcal{E}_{\infty, \bar{n}}\right)_{\Upsilon_{m}^{\prime}} \rightarrow \mathcal{E}_{K_{m, \bar{n}}^{\prime}}\right)$. For all $n \geq \bar{n}$ there is $m \in N$ such that the image of $\Upsilon_{n}$ in $\left(\bar{\Upsilon} / \bar{\Upsilon}_{\bar{n}}\right) \times \Upsilon^{\prime}$ is $\Upsilon_{m}^{\prime}$. Then $K_{n, \bar{n}}=K_{m, \bar{n}}^{\prime},\left(\mathcal{E}_{\infty, \bar{n}}\right) \Upsilon_{n}=\left(\mathcal{E}_{\infty, \bar{n}}\right) \Upsilon_{m}^{\prime}$ and $\left(\mathcal{U}_{\infty, \bar{n}}\right) \Upsilon_{n}=\left(\mathcal{U}_{\infty, \bar{n}}\right)_{\Upsilon_{m}^{\prime}}$, from which we deduce Lemma 3.2.

LEMMA 3.3. There is a natural number $\bar{m}_{0} \in N$ such that, for all nonnegative integers $\bar{n} \leq n, p^{c_{\bar{n}}}\left(\gamma_{\delta^{\prime}}^{\prime}-1\right)\left(\bar{\gamma}_{\bar{m}_{0}}-1\right)^{2}$ annihilates $\operatorname{Ker}\left(\pi_{n, \bar{n}}^{\mathcal{E}}\right)$ and $\operatorname{Cok}\left(\pi_{n, \bar{n}}^{\mathcal{E}}\right)$.

ProOF. Let us momentaneously fix $\bar{n}$. From Lemma 3.1, we deduce an isomorphism $\operatorname{Cok}\left(\pi_{\infty, \bar{n}}^{\mathcal{E}}\right) \simeq \operatorname{Ker}\left(\pi_{\infty, \bar{n}}^{\mathcal{U} / \mathcal{E}}\right)$ and an exact sequence

$$
\operatorname{Ker}\left(\pi_{\infty, \bar{n}}^{\mathcal{U} / \mathcal{E}}\right)^{\Upsilon_{n}} \longrightarrow\left(\mathcal{E}_{\infty}\right)_{\Gamma_{n, \bar{n}}} \longrightarrow\left(\mathcal{E}_{\infty, \bar{n}}\right)_{\Upsilon_{n}} \longrightarrow \operatorname{Ker}\left(\pi_{\infty, \bar{n}}^{\mathcal{U} / \mathcal{E}}\right) \Upsilon_{n} \longrightarrow 0
$$

By Proposition 2.5 (iii) we have the commutative diagram with exact rows:

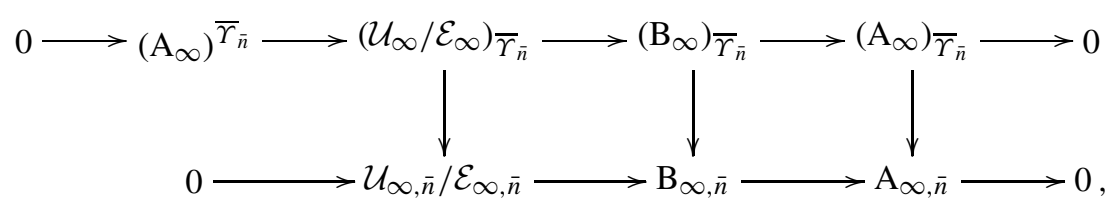

from which we deduce the exact sequence

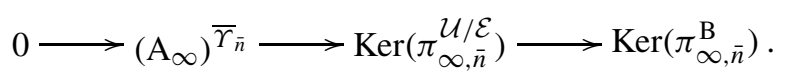

Since $\mathrm{A}_{\infty}$ is Noetherian, there is $\bar{m}_{0} \in N$ such that $\left(\mathrm{A}_{\infty}\right)^{\bar{\Upsilon}_{\bar{m}}} \subseteq\left(\mathrm{A}_{\infty}\right)^{\bar{\Upsilon}_{\bar{m}_{0}}}$ for all $\bar{m} \in N$. We can choose $\bar{m}_{0}$ such that $\bar{\gamma}_{\bar{m}_{0}} \in \bar{D}$. Then by Theorem 2.4 (ii),

$$
\bar{\gamma}_{\bar{m}_{0}}-1 \text { annihilates } \operatorname{Ker}\left(\pi_{\infty, \bar{m}}^{\mathrm{B}}\right) \text { and }\left(\mathrm{A}_{\infty}\right)^{\bar{\Upsilon}_{\bar{m}}} \text { for all } \bar{m} \in N .
$$

By (3.8), $\left(\bar{\gamma}_{\bar{m}_{0}}-1\right)^{2}$ annihilates $\operatorname{Ker}\left(\pi_{\infty, \bar{n}}^{\mathcal{U} / \mathcal{E}}\right)$. From (3.6) and Lemma 3.2, we deduce Lemma 3.3.

Proposition 3.4. There is a finite set T, a family $\left(n_{t}\right)_{t \in T} \in N^{T}$, and a pseudoisomorphism of $\Lambda_{\infty, \chi}$-modules

$$
\Theta_{\chi}: \mathcal{E}_{\infty, \chi} \longrightarrow \Lambda_{\infty, \chi} \oplus \bigoplus_{t \in T}\left(\Lambda_{\infty, \chi} / \mathfrak{u}_{\chi}^{n_{t}}\right),
$$

where $\mathfrak{u}_{\chi}$ is a uniformizer of $\boldsymbol{Z}_{p}[\chi]$. 
Proof. By Theorem 2.1, $e_{\psi}\left(\boldsymbol{Q}_{p} \otimes_{\boldsymbol{Z}_{p}} \mathcal{U}_{\infty}\right)$ is a torsion-free rank one $e_{\psi}\left(\boldsymbol{Q}_{p} \otimes_{\boldsymbol{Z}_{p}} \Lambda_{\infty}\right)$ module. From Theorem 2.4 (i) and (3.2), we deduce that the $\Lambda_{\infty, \chi}$-module $\mathcal{E}_{\infty, \chi}$ is of rank 1 and that its torsion is annihilated by a power of $p$.

Let pr : $\Lambda_{\infty, \chi} \oplus \bigoplus_{t \in T}\left(\Lambda_{\infty, \chi} / \mathfrak{u}_{\chi}^{n_{t}}\right) \rightarrow \Lambda_{\infty, \chi}$ be the canonical projection. For all nonnegative integers $\bar{n} \leq n$, let $\Theta_{n, \bar{n}, \chi}:\left(\mathcal{E}_{\infty, \chi}\right)_{\Gamma_{n, \bar{n}}} \rightarrow \boldsymbol{Z}_{p}[\chi]\left[\Gamma / \Gamma_{n, \bar{n}}\right]$ be the map obtained from pr $\Theta_{\chi}$ by taking the quotients. Let $h_{\chi}$ be a generator of $\operatorname{char}_{\Lambda_{\infty, \chi}}\left(\mathcal{E}_{\infty} / \mathcal{C}_{\infty}\right)_{\chi}$. As in [1, proof of Lemma 3.5] or [19, Lemma 6.3], one can deduce from Lemma 3.3 that $p^{2 c_{\bar{n}}}\left(\gamma_{\delta^{\prime}}^{\prime}-1\right)^{2}\left(\bar{\gamma}_{\bar{m}_{0}}-1\right)^{4}$ annihilates $\operatorname{Ker}\left(\pi_{n, \bar{n}}^{\mathcal{E}_{\chi}}\right)$ and $\operatorname{Cok}\left(\pi_{n, \bar{n}}^{\mathcal{E}_{\chi}}\right)$. Then one easily verifies that the map

$$
\vartheta_{n, \bar{n}, \chi}: \mathcal{E}_{n, \bar{n}, \chi} \rightarrow \boldsymbol{Z}_{p}[\chi]\left[\Gamma / \Gamma_{n, \bar{n}}\right], \quad x \mapsto p^{2 c_{\bar{n}}}\left(\gamma_{\delta^{\prime}}^{\prime}-1\right)^{2}\left(\bar{\gamma}_{\bar{m}_{0}}-1\right)^{4} \Theta_{n, \bar{n}, \chi}(\tilde{x})
$$

is well defined, where $\tilde{x} \in\left(\mathcal{E}_{\infty, \chi}\right) \Gamma_{n, \bar{n}}$ verifies $\pi_{n, \bar{n}}^{\mathcal{E}_{\chi}}(\tilde{x})=p^{2 c_{\bar{n}}}\left(\gamma_{\delta^{\prime}}^{\prime}-1\right)^{2}\left(\bar{\gamma}_{\bar{m}_{0}}-1\right)^{4} x$.

4. Elliptic units. For two $Z$-lattices $L$ and $L^{\prime}$ of $\boldsymbol{C}$ such that $L \subseteq L^{\prime}$ and $\left[L^{\prime}: L\right]$ is prime to 6 , we denote by $z \mapsto \psi\left(z ; L, L^{\prime}\right)$ the elliptic function defined in [14]. For a nonzero proper ideal $\mathfrak{m}$ of $\mathcal{O}_{k}$, and a nonzero ideal $\mathfrak{a}$ of $\mathcal{O}_{k}$ prime to $6 \mathfrak{m}$, Robert proved that $\psi\left(1 ; \mathfrak{m}, \mathfrak{a}^{-1} \mathfrak{m}\right) \in \mathrm{H}(\mathfrak{m})$, where $\mathrm{H}(\mathfrak{m})$ is the ray class field modulo $\mathfrak{m}$ of $k$. If $\varphi_{\mathfrak{m}}(1) \in \mathrm{H}(\mathfrak{m})^{\times}$ is the Robert-Ramachandra invariant, as defined in [12, p. 15], we have by [13, Corollaire 1.3, (iii)]

$$
\psi\left(1 ; \mathfrak{m}, \mathfrak{a}^{-1} \mathfrak{m}\right)^{12 \varrho(\mathfrak{m})}=\varphi_{\mathfrak{m}}(1)^{\mathrm{N}(\mathfrak{a})-(\mathfrak{a}, \mathrm{H}(\mathfrak{m}) / k)},
$$

where $\varrho(\mathfrak{m})$ is the positive generator of $\mathfrak{m} \cap \boldsymbol{Z}, \mathrm{N}(\mathfrak{a}):=\#\left(\mathcal{O}_{k} / \mathfrak{a}\right)$, and $(\mathfrak{a}, \mathrm{H}(\mathfrak{m}) / k)$ is the Artin automorphism of $\mathrm{H}(\mathfrak{m}) / k$ defined by $\mathfrak{a}$. Let $\mathrm{S}(\mathfrak{m})$ be the set of maximal ideals of $\mathcal{O}_{k}$ which divide $\mathfrak{m}$. If we denote by $w_{\mathfrak{m}}$ the number of roots of unity of $k$ which are congruent to 1 modulo $\mathfrak{m}$, and if we write $w_{k}$ for the number of roots of unity of $k$, then by $[13$, p. 21 , $\left(\right.$ iv $\left.^{\prime}\right)$ ], we have

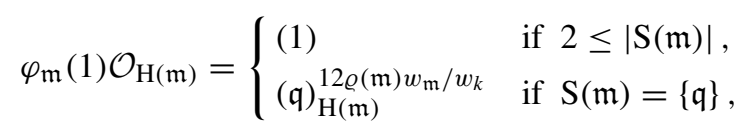

where $(\mathfrak{q})_{\mathrm{H}(\mathfrak{m})}$ is the product of the prime ideals of $\mathcal{O}_{\mathrm{H}(\mathfrak{m})}$ which lie above $\mathfrak{q}$. Moreover, if $\mathfrak{a}$ is prime to $6 \mathfrak{m q}$, then by $[13$, p. 21 , (ii'-1)] we have

$$
\mathrm{N}_{\mathrm{H}(\mathfrak{m} \mathfrak{q}) / \mathrm{H}(\mathfrak{m})}\left(\varphi_{\mathfrak{m} \mathfrak{q}}(1)\right)= \begin{cases}\varphi_{\mathfrak{m}}(1)^{\varrho(\mathfrak{m} \mathfrak{q}) \varrho(\mathfrak{m})^{-1}\left(1-(\mathfrak{q}, \mathrm{H}(\mathfrak{m}) / k)^{-1}\right)} & \text { if } \mathfrak{q} \nmid \mathfrak{m} \\ \varphi_{\mathfrak{m}}(1)^{\varrho(\mathfrak{m} \mathfrak{q}) \varrho(\mathfrak{m})^{-1}} & \text { if } \mathfrak{q} \mid \mathfrak{m} .\end{cases}
$$

Definition 4.1. Let $F \subset \boldsymbol{C}$ be a finite abelian extension of $k$. We write $\Psi_{F}$ for the $\boldsymbol{Z}[\mathrm{Gal}(F / k)]$-submodule of $F^{\times}$generated by $\mu(F)$ and by all the norms $\mathrm{N}_{\mathrm{H}(\mathfrak{m}) / \mathrm{H}(\mathfrak{m}) \cap F}\left(\psi\left(1 ; \mathfrak{m}, \mathfrak{a}^{-1} \mathfrak{m}\right)\right)$, where $\mathfrak{m}$ is a nonzero proper ideal of $\mathcal{O}_{k}$ and $\mathfrak{a}$ is any nonzero ideal of $\mathcal{O}_{k}$ prime to $6 \mathrm{~m}$. Then we define the groups $C_{F}:=\mathcal{O}_{F}^{\times} \cap \Psi_{F}$ and $\mathcal{C}_{F}:=Z_{p} \otimes_{Z} C_{F}$.

For any abelian extension $F$ of $k$, we set $\tilde{\mathcal{K}}(F):=\lim _{F^{\prime}}\left(\boldsymbol{Q}_{p} \otimes_{\boldsymbol{Z}}\left(F^{\prime}\right)^{\times}\right)$, where the limit is taken over all finite subextensions $F^{\prime} \subseteq F$ of $k$ with respect to the norm maps. We fix a nonzero ideal $\mathfrak{f}$ of $\mathcal{O}_{k}$, prime to $(p)$, such that $K_{\infty} \subseteq \mathrm{H}\left(\mathfrak{f} p^{\infty}\right)$, where 
$\mathrm{H}\left(\mathfrak{f} p^{\infty}\right):=\bigcup_{n \in N} \mathrm{H}\left(\mathfrak{f} p^{n}\right)$. For any ideal $\mathfrak{g}$ of $\mathcal{O}_{k}$ dividing $\mathfrak{f}$, and for any $(n, \bar{n}) \in N^{2}$, we set

$$
\varphi_{\mathfrak{g}, n, \bar{n}}:=\frac{1}{12 \varrho\left(\mathfrak{g p}^{n+1} \overline{\mathfrak{p}}^{\bar{n}+1}\right)} \otimes \varphi_{\mathfrak{g p}^{n+1} \overline{\mathfrak{p}}^{\bar{n}+1}}(1)
$$

in $\boldsymbol{Q}_{p} \otimes_{\mathbf{Z}} \mathrm{H}\left(\mathfrak{g} \mathfrak{p}^{n+1} \overline{\mathfrak{p}}^{\bar{n}+1}\right)^{\times}$. Then by (4.3) $\varphi_{\mathfrak{g}}:=\left(\varphi_{\mathfrak{g}, n, \bar{n}}\right)_{(n, \bar{n}) \in N^{2}}$ is well defined in

$$
\tilde{\mathcal{K}}\left(\mathrm{H}\left(\mathfrak{g} p^{\infty}\right)\right)=\lim _{n, \bar{n}}\left(\boldsymbol{Q}_{p} \otimes_{\boldsymbol{Z}} \mathrm{H}\left(\mathfrak{g p} \mathfrak{p}^{n+1} \overline{\mathfrak{p}}^{\bar{n}+1}\right)^{\times}\right)
$$

Since $\lim _{\leftarrow}{ }_{\digamma K_{\infty}} \mu(F)=0$, where the limit is taken over all finite subextensions $F \subset K_{\infty}$ of $K$ with respect to the norm maps, we deduce that the natural map $\mathcal{C}_{\infty} \rightarrow \tilde{\mathcal{K}}_{\infty}$ is injective, where $\mathcal{C}_{\infty}$ and $\tilde{\mathcal{K}}_{\infty}$ are defined in accordance with our conventions (Section 1). Proceeding as in [20, Lemma 2.7], one can show that as a $\Lambda_{\infty}$-submodule of $\tilde{\mathcal{K}}_{\infty}, \mathcal{C}_{\infty}$ is generated by all the elements

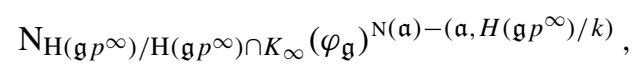

where $\mathfrak{g}$ is an ideal of $\mathcal{O}_{k}$ dividing $\mathfrak{f}$ and $\mathfrak{a}$ is an ideal of $\mathcal{O}_{k}$ prime to $6 p \mathfrak{g}$. We set

$$
\mathcal{Q}:=\boldsymbol{Q}_{p}(\zeta)
$$

where $\zeta$ is a primitive $\left[\mathrm{H}\left(\mathfrak{f} p^{\infty}\right): k_{\infty}\right]$-th root of unity. Let us also denote by $\chi_{\mathfrak{f}}$ the character of $\mathrm{Gal}\left(\mathrm{H}\left(\mathfrak{f} p^{\infty}\right) / k_{\infty}\right)$ obtained from $\chi$ by inflation.

LEMMA 4.2. Let $\mathfrak{f}_{\chi}$ be the smallest ideal of $\mathcal{O}_{k}$ dividing $\mathfrak{f}$ such that $\chi_{\mathfrak{f}}$ is trivial on the Galois group $\operatorname{Gal}\left(\mathrm{H}\left(\mathfrak{f} p^{\infty}\right) / K_{\infty} \cap \mathrm{H}\left(\mathfrak{f}_{\chi} p^{\infty}\right)\right)$. Then in $\mathcal{Q} \otimes_{Z_{p}} \tilde{\mathcal{K}}_{\infty}$, we have

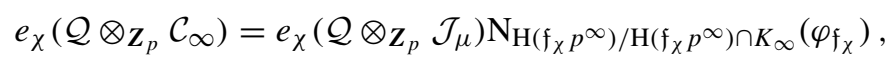

where $\mathcal{J}_{\mu}$ is the annihilator of $\mu_{p}\left(K_{\infty}\right)$ over $\Lambda_{\infty}$.

Proof. Let $\mathfrak{g}$ be an ideal of $\mathcal{O}_{k}$ dividing $\mathfrak{f}$. Let us first assume that $\mathfrak{f}_{\chi} \mid \mathfrak{g}$. We set $a:=$ $\left[\mathrm{H}\left(\mathfrak{g} p^{\infty}\right) \cap K_{\infty}: \mathrm{H}\left(\mathfrak{f}_{\chi} p^{\infty}\right) \cap K_{\infty}\right]$, and for any prime $l$ dividing $\mathfrak{f}$ and not dividing $\mathfrak{f}_{\chi}$, we set $\sigma_{l}:=\left(l, \mathrm{H}\left(\mathfrak{f}_{\chi} p^{\infty}\right) / k\right)^{-1}$. Since $\chi_{\mathfrak{f}}$ is trivial on $\operatorname{Gal}\left(\mathrm{H}\left(\mathfrak{f} p^{\infty}\right) / K_{\infty} \cap \mathrm{H}\left(\mathfrak{f}_{\chi} p^{\infty}\right)\right)$ we obtain

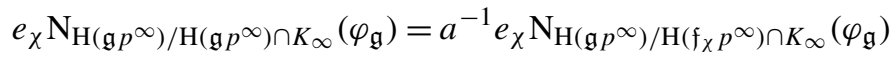

$$
\begin{aligned}
& =\left(\prod_{l}\left(1-\sigma_{l}\right)\right) a^{-1} e_{\chi} \mathrm{N}_{\mathrm{H}\left(\mathfrak{f}_{\chi} p^{\infty}\right) / \mathrm{H}\left(\mathfrak{f}_{\chi} p^{\infty}\right) \cap K_{\infty}}\left(\varphi_{\mathfrak{f}_{\chi}}\right),
\end{aligned}
$$

where the product is over all primes $l$ dividing $\mathfrak{g}$ but not dividing $\mathfrak{f}_{\chi}$. Now assume that $\mathfrak{f}_{\chi} \nmid \mathfrak{g}$. Then $\chi_{\mathfrak{f}}$ is not trivial on $\operatorname{Gal}\left(\mathrm{H}\left(\mathfrak{f} p^{\infty}\right) / K_{\infty} \cap \mathrm{H}\left(\mathfrak{g} p^{\infty}\right)\right)$, and

$$
e_{\chi} \mathrm{N}_{\mathrm{H}\left(\mathfrak{g} p^{\infty}\right) / \mathrm{H}\left(\mathfrak{g} p^{\infty}\right) \cap K_{\infty}}\left(\varphi_{\mathfrak{g}}\right)=\left[\mathrm{H}\left(\mathfrak{f} p^{\infty}\right): \mathrm{H}\left(\mathfrak{g} p^{\infty}\right)\right]^{-1} e_{\chi} \mathrm{N}_{\mathrm{H}\left(\mathfrak{f} p^{\infty}\right) / \mathrm{H}\left(\mathfrak{g} p^{\infty}\right) \cap K_{\infty}}\left(\varphi_{\mathfrak{g}}\right)=0 .
$$

Then the lemma follows from (4.4), (4.1) and the description of $\mathcal{J}_{\mu}$ given in [18, IV, Lemme $1.1]$.

For all ideal $\mathfrak{g}$ of $\mathcal{O}_{k}$ dividing $\mathfrak{f p}^{m}$ for some $m \in N$, and for all $n \in N$, we set

$$
\varphi_{\mathfrak{g}, n}^{\prime}:=\frac{1}{12 \varrho\left(\mathfrak{g p}^{n+1}\right)} \otimes \varphi_{\mathfrak{g p}^{n+1}}(1)
$$


in $\boldsymbol{Q}_{p} \otimes_{\boldsymbol{Z}} \mathrm{H}\left(\mathfrak{g p}^{n+1}\right)^{\times}$. Then $\varphi_{\mathfrak{g}}^{\prime}:=\left(\varphi_{\mathfrak{g}, n}^{\prime}\right)_{n \in N}$ is well defined in $\left.\tilde{\mathcal{K}}\left(\mathrm{H}(\mathfrak{g p})^{\infty}\right)\right)=\lim _{n}$ $\left(\boldsymbol{Q}_{p} \otimes_{\boldsymbol{Z}} \mathrm{H}\left(\mathfrak{g p}^{n+1}\right)^{\times}\right)$.

REMARK 4.3. It is well known that Stark units can be constructed by means of elliptic units (see [13] for a precise statement). Then it is an easy matter to verify that $\mathcal{C}_{\infty, 0}=\overline{S t}_{\infty}$, where $\overline{S t}_{\infty}$ is the module of Stark units for $K_{\infty, 0}$, as defined in [7]. Then from [7, Theorem 3.2 and Proposition 2.1] we deduce that $\mathcal{C}_{\infty, 0}$ is finitely generated over $\Lambda_{\gamma^{\prime}}$ of $\operatorname{rank}[K: k]$, and the rank of $\mathcal{C}_{\infty, 0, \chi}$ over $\boldsymbol{Z}_{p}[\chi]\left[\left[\Upsilon^{\prime}\right]\right]$ is 1 .

We arbitrarily lift $\Gamma$ into a subgroup $\Gamma_{\mathfrak{f}}$ of $\operatorname{Gal}\left(\mathrm{H}\left(\mathfrak{f} p^{\infty}\right) / k\right)$. We denote by $\bar{\Upsilon}_{\mathfrak{f}}$ the subgroup corresponding to $\bar{\Upsilon}$, and we denote by $\bar{J}_{\mathfrak{f}}$ the inertia group of $\overline{\mathfrak{p}}$ in $\mathrm{H}\left(\mathfrak{f} p^{\infty}\right) / k$. Let $N \in N$ be such that $\bar{J}_{\mathfrak{f}} \cap \Gamma_{\mathfrak{f}}=\bar{\Upsilon}_{\mathfrak{f}}^{p^{N}}$. For any module $M$ over any ring $R$, we denote by $\operatorname{Nul}_{R}(M)$ the annihilator of $M$ over $R$. As a $\Lambda_{\infty}$-submodule of $\tilde{\mathcal{K}}_{\infty, 0}:=\tilde{\mathcal{K}}\left(K_{\infty, 0}\right), \mathcal{C}_{\infty, 0}$ is generated by all the elements

$$
\left.\mathrm{N}_{\mathrm{H}(\mathfrak{g p}} \infty\right) / \mathrm{H}\left(\mathfrak{g p} \mathfrak{p}^{\infty}\right) \cap K_{\infty, 0}\left(\varphi_{\mathfrak{g}}^{\prime}\right)^{\alpha},
$$

where $\mathfrak{g}$ is an ideal of $\mathcal{O}_{k}$ dividing $\mathfrak{f p}^{N+1}$ and $\alpha \in \operatorname{Nul}_{\Lambda_{\infty}}\left(\mu_{p^{\infty}}\left(K_{\infty, 0}\right)\right)$ if $\mathfrak{g} \neq 1, \alpha \in$ $\operatorname{Nul}_{\Lambda_{\infty}}\left(\mu_{p^{\infty}}\left(K_{\infty, 0}\right)\right) \cap \mathcal{J}_{\infty}$ if $\mathfrak{g}=1$ (see [20, Lemma 2.7]). We denote by $\bar{J}$ the inertia group of $\overline{\mathfrak{p}}$ in $K_{\infty} / k$, and by $\tilde{\chi}$ the character of $G_{\infty}$ obtained from $\chi$ by inflation.

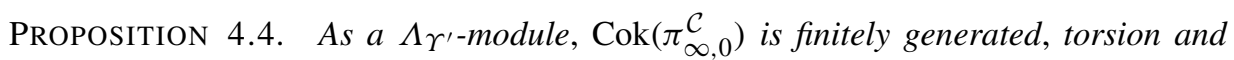
annihilated by $\mathcal{J}_{\bar{D}}$. Its characteristic ideal over $\Lambda_{\gamma^{\prime}}$ is prime to $p$. Moreover, we have

$$
e_{\chi}\left(\mathcal{Q} \otimes \boldsymbol{Z}_{p} \operatorname{Cok}\left(\pi_{\infty, 0}^{\mathcal{C}}\right)\right)= \begin{cases}\left(\mathcal{Q} \otimes_{\boldsymbol{Z}_{p}} \Lambda_{\Upsilon^{\prime}}\right) / \mathcal{J}_{\bar{D}}\left(\mathcal{Q} \otimes_{\boldsymbol{Z}_{p}} \Lambda_{\Upsilon^{\prime}}\right) & \text { if } \tilde{\chi} \neq 1 \text { is trivial on } \bar{J}, \\ 0 & \text { if } \tilde{\chi} \text { is not trivial on } \bar{J} \\ \mathcal{J}_{\infty}\left(\mathcal{Q} \otimes_{\boldsymbol{Z}_{p}} \Lambda_{\Upsilon^{\prime}}\right) / \mathcal{J}_{\bar{D}}\left(\mathcal{Q} \otimes_{\boldsymbol{Z}_{p}} \Lambda_{\Upsilon^{\prime}}\right) & \text { if } \chi=1 .\end{cases}
$$

ProOF. Since $\mu\left(K_{\infty, 0}\right)$ is finite, calculations which are similar to those done in the proof of Lemma 4.2 give us the following equalities:

$$
\begin{aligned}
& e_{\chi}\left(\mathcal{Q} \otimes Z_{p} \mathcal{C}_{\infty, 0}\right)
\end{aligned}
$$

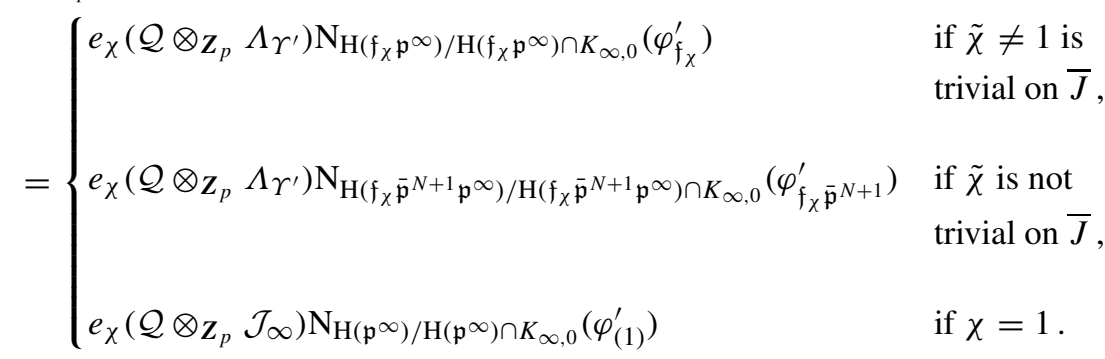

On the other hand, for all ideals $\mathfrak{g}$ dividing $\mathfrak{f}$, one has

$$
\mathrm{N}_{K_{\infty} / K_{\infty, 0}}\left(\mathrm{~N}_{\mathrm{H}\left(\mathfrak{g} p^{\infty}\right) / \mathrm{H}\left(\mathfrak{g} p^{\infty}\right) \cap K_{\infty}}\left(\varphi_{\mathfrak{g}}\right)\right)=\mathrm{N}_{\mathrm{H}\left(\mathfrak{g} \overline{\mathfrak{p}}^{N+1} \mathfrak{p}^{\infty}\right) / \mathrm{H}\left(\mathfrak{g} \overline{\mathfrak{p}}^{N+1} \mathfrak{p}^{\infty}\right) \cap K_{\infty, 0}}\left(\varphi_{\mathfrak{g}^{\prime} \overline{\mathfrak{p}}^{N+1}}^{\prime}\right)
$$


where the norm maps are defined in accordance with our conventions (Section 1). From (4.8) and Lemma 4.2, one can calculate the image of $e_{\chi}\left(\mathcal{Q} \otimes \boldsymbol{Z}_{p} \mathcal{C}_{\infty}\right)$ in $\mathcal{Q} \otimes \mathbf{Z}_{p} \mathcal{C}_{\infty, 0}$, and then derive from (4.7) the expressions of $e_{\chi}\left(\mathcal{Q} \otimes z_{p} \operatorname{Cok}\left(\pi_{\infty, 0}^{\mathcal{C}}\right)\right)$ given in Proposition 4.4. From (4.8) we deduce that, for all ideal $\mathfrak{g}$ dividing $\mathfrak{f}$ and for all $\alpha \in \operatorname{Nul}_{\Lambda_{\infty}}\left(\mu_{p} \infty\left(K_{\infty, 0}\right)\right)$,

$$
\mathrm{N}_{\mathrm{H}\left(\mathfrak{g p} \mathfrak{p}^{\infty}\right) / \mathrm{H}\left(\mathfrak{g} \mathfrak{p}^{\infty}\right) \cap K_{\infty, 0}}\left(\varphi_{\mathfrak{g}}^{\prime}\right)^{\left(1-\sigma_{\overline{\mathfrak{p}}}\right) \alpha} \in \operatorname{Im}\left(\mathcal{C}_{\infty}\right),
$$

where $\operatorname{Im}\left(\mathcal{C}_{\infty}\right)$ is the image of $\mathcal{C}_{\infty}$ in $\mathcal{C}_{\infty, 0}$, and $\sigma_{\overline{\mathfrak{p}}}:=\left(\overline{\mathfrak{p}}, \mathrm{H}\left(\mathfrak{g} p^{\infty}\right) / k\right)^{-1}$. We deduce that $\mathcal{J}_{\bar{D}}$ annihilates $\operatorname{Cok}\left(\pi_{\infty, 0}^{\mathcal{C}}\right)$. Then $\operatorname{Cok}\left(\pi_{\infty, 0}^{\mathcal{C}}\right)$ is torsion over $\Lambda_{\Upsilon^{\prime}}$, and finitely generated by Remark 4.3. In order to verify that $\operatorname{char}_{\Lambda_{\gamma^{\prime}}}\left(\operatorname{Cok}\left(\pi_{\infty, 0}^{\mathcal{C}}\right)\right)$ is prime to $p$, we just have to notice that $\Lambda_{\Upsilon^{\prime}} / \mathcal{J}_{\bar{D}} \Lambda_{\Upsilon^{\prime}} \simeq \boldsymbol{Z}_{p}[\Gamma / \bar{D} \bar{\Upsilon}]$ is finitely generated over $\boldsymbol{Z}_{p}$.

LEMMA 4.5. Let $M$ be a finitely generated torsion $\Lambda_{\Gamma}$-module, such that $M_{\bar{\gamma}}$ is torsion over $\Lambda_{\Upsilon^{\prime}}$ and $\operatorname{char}_{\Lambda_{\Upsilon^{\prime}}} M_{\bar{\Upsilon}}$ is prime to $p$. Then $\operatorname{char}_{\Lambda_{\Gamma}} M$ is prime to $p$.

PRoOF. Let $\left(\mathfrak{r}_{t}\right)_{t \in T}$ be a finite family of height 1 prime ideals of $\Lambda_{\Gamma}$, and $\left(m_{t}\right)_{t \in T} \in N^{T}$ be such that $M^{\prime}:=\bigoplus_{t \in T} \Lambda_{\Gamma} / \mathfrak{r}_{t}^{m_{t}}$ is pseudo-isomorphic to $M$. Then there exists an exact sequence

$$
0 \longrightarrow M^{\prime} \longrightarrow M \longrightarrow P \longrightarrow 0
$$

where $P$ is a pseudo-null $\Lambda_{\Gamma}$-module. Then we have an exact sequence

$$
P^{\bar{\Upsilon}} \longrightarrow M_{\bar{\Upsilon}}^{\prime} \longrightarrow M_{\bar{\Upsilon}} \longrightarrow P_{\bar{\Upsilon}} \longrightarrow 0
$$

Moreover, by [11, Lemme 4 (2)] and since $M_{\bar{\Upsilon}}$ is torsion over $\Lambda_{\Upsilon^{\prime}}, P^{\bar{\Upsilon}}$ and $P_{\bar{\Upsilon}}$ are both finitely generated and torsion over $\Lambda_{\Upsilon^{\prime}}$, and

$$
\operatorname{char}_{\Lambda_{\Upsilon^{\prime}}}\left(P^{\bar{\Upsilon}}\right)=\operatorname{char}_{\Lambda_{\Upsilon^{\prime}}}(P \bar{\Upsilon}) .
$$

From (4.10) and (4.11), and since $\operatorname{char}_{\Lambda_{\Upsilon^{\prime}}} M_{\bar{\Upsilon}}$ is prime to $p$, we deduce that $\operatorname{char}_{\Lambda_{\gamma^{\prime}}}\left(M_{\bar{\gamma}}^{\prime}\right)$ is prime to $p$. From the equality $M^{\prime}=\bigoplus_{t \in T} \Lambda_{\Gamma} / \mathfrak{r}_{t}^{m_{t}}$, we deduce that $\operatorname{char}_{\Lambda_{\Gamma}}\left(M^{\prime}\right)$ is prime to $p$. Since $M$ and $M^{\prime}$ are pseudo-isomorphic, the lemma follows.

THEOREM 4.6. The following properties hold.

(i) $\mathcal{C}_{\infty}$ is a torsion-free finitely generated $\Lambda_{\Gamma}$-module of rank $[K: k]$.

(ii) $\mathcal{U}_{\infty} / \mathcal{C}_{\infty}, \mathcal{U}_{\infty} / \mathcal{E}_{\infty}$ and $\mathcal{E}_{\infty} / \mathcal{C}_{\infty}$ are finitely generated and torsion over $\Lambda_{\Gamma}$.

(iii) $\operatorname{char}_{\Lambda_{\Gamma}}\left(\mathcal{U}_{\infty} / \mathcal{C}_{\infty}\right)$, $\operatorname{char}_{\Lambda_{\Gamma}}\left(\mathcal{U}_{\infty} / \mathcal{E}_{\infty}\right)$ and $\operatorname{char}_{\Lambda_{\Gamma}}\left(\mathcal{E}_{\infty} / \mathcal{C}_{\infty}\right)$ are prime to $p$ and $\bar{\gamma}-1$.

(iv) $\mathcal{U}_{\infty, 0} / \mathcal{C}_{\infty, 0}, \mathcal{U}_{\infty, 0} / \mathcal{E}_{\infty, 0}$, and $\mathcal{E}_{\infty, 0} / \mathcal{C}_{\infty, 0}$ are finitely generated and torsion over $\Lambda_{\Upsilon^{\prime}}$.

PROOF. The module $\mathcal{C}_{\infty}$ is a submodule of $\mathcal{U}_{\infty}$, hence it is torsion-free and finitely generated over $\Lambda_{\Gamma}$, of rank at most [K:k] by Theorem 2.1. By Lemma 4.2, we see that $e_{\chi}\left(\mathcal{Q} \otimes \mathbf{Z}_{p} \mathcal{C}_{\infty}\right)$ has rank 1 over $\mathcal{Q} \otimes \mathbf{Z}_{p} \Lambda_{\Gamma}$, or is zero. But we know from Remark 4.3 that $e_{\chi}\left(\mathcal{Q} \otimes z_{p} \mathcal{C}_{\infty, 0}\right)$ has rank 1 over $\mathcal{Q} \otimes z_{p} \Lambda_{\Upsilon^{\prime}}$. Hence from Proposition 4.4 we see that $e_{\chi}\left(\mathcal{Q} \otimes \boldsymbol{Z}_{p} \mathcal{C}_{\infty}\right)$ has rank 1 over $\mathcal{Q} \otimes \boldsymbol{Z}_{p} \Lambda_{\Gamma}$. Considering all irreducible $\boldsymbol{C}_{p}$-characters of $G$, 
we deduce (i). Then (ii) follows from (i) and Theorem 2.1. We have the exact sequence

$$
0 \longrightarrow \operatorname{Cok}\left(\pi_{\infty, 0}^{\mathcal{C}}\right) \longrightarrow \mathcal{U}_{\infty, 0} / \operatorname{Im}\left(\mathcal{C}_{\infty}\right) \longrightarrow \mathcal{U}_{\infty, 0} / \mathcal{C}_{\infty, 0} \longrightarrow 0
$$

where $\operatorname{Im}\left(\mathcal{C}_{\infty}\right)$ is the image of $\mathcal{C}_{\infty}$ in $\mathcal{C}_{\infty, 0}$. But $\operatorname{char}_{\Lambda_{\gamma^{\prime}}}\left(\operatorname{Cok}\left(\pi_{\infty, 0}^{\mathcal{C}}\right)\right)$ is prime to $p$ by Proposition 4.4, and char $\Lambda_{\Lambda^{\prime}}\left(\mathcal{U}_{\infty, 0} / \mathcal{C}_{\infty, 0}\right)$ is prime to $p$ by [20, Theorem 1.1] and Gillard's result $\left[3,3.4\right.$. Théorème]. Hence $\operatorname{char}_{\Lambda_{\Upsilon^{\prime}}}\left(\mathcal{U}_{\infty, 0} / \operatorname{Im}\left(\mathcal{C}_{\infty}\right)\right)$ is prime to $p$. By Lemma 2.2, we have the following commutative diagram with exact rows:

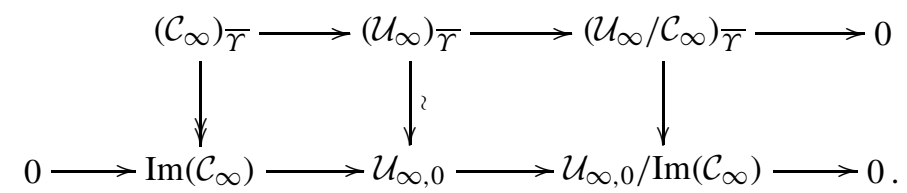

A diagram chase shows that the last vertical arrow is an isomorphism. Then $\operatorname{char}_{\Lambda_{\Gamma}}\left(\mathcal{U}_{\infty} / \mathcal{C}_{\infty}\right)$ is prime to $p$ by Lemma 4.5 , and by [11, Lemme $4(1)] \operatorname{char}_{\Lambda_{\Gamma}}\left(\mathcal{U}_{\infty} / \mathcal{C}_{\infty}\right)$ is prime to $\bar{\gamma}-1$. Since $\mathcal{E}_{\infty} / \mathcal{C}_{\infty}$ and $\mathcal{U}_{\infty} / \mathcal{E}_{\infty}$ are respectively a submodule and a quotient of $\mathcal{U}_{\infty} / \mathcal{C}_{\infty}$, (iii) is entirely proved. (iv) is a corollary of Remark 4.3 (see [20, Proposition 4.2] or [19, Proposition 3.1] for more details).

PROPOSITION 4.7. We have $\mathcal{Q} \otimes \mathbf{z}_{p} \operatorname{Ker}\left(\pi_{\infty, 0}^{\mathcal{C}}\right)=0$.

Proof. Since $\mu\left(K_{\infty, 0}\right)$ is finite, $\Lambda_{\Upsilon^{\prime}} / \mathcal{J}_{\mu} \Lambda_{\Upsilon^{\prime}}$ is finite. Then from Lemma 4.2 and the proof of Theorem 4.6 (i), we deduce that $e_{\chi}\left(\mathcal{Q} \otimes \mathbf{z}_{p} \mathcal{C}_{\infty}\right) \bar{\Upsilon}$ is a free rank one $\mathcal{Q} \otimes \mathbf{Z}_{p} \Lambda_{\Upsilon^{\prime}}$ module. Considering all irreducible $\boldsymbol{C}_{p}$-characters of $G$, we see that $\left(\mathcal{Q} \otimes \boldsymbol{Z}_{p} \mathcal{C}_{\infty}\right) \bar{\gamma}$ is a free $\mathcal{Q} \otimes \boldsymbol{Z}_{p} \Lambda_{\Upsilon^{\prime}}$-module of rank $[K: k]$. But $\mathcal{Q} \otimes \boldsymbol{z}_{p} \mathcal{C}_{\infty, 0}$ has rank $[K: k]$ over $\mathcal{Q} \otimes \boldsymbol{Z}_{p} \Lambda_{\Upsilon^{\prime}}$ by Remark 4.3, hence by Proposition 4.4 we must have $\mathcal{Q} \otimes \mathbf{z}_{p} \operatorname{Ker}\left(\pi_{\infty, 0}^{\mathcal{C}}\right)=0$.

Let $h_{\chi}$ be a generator of $\operatorname{char}_{\Lambda_{\infty, \chi}}\left(\mathcal{E}_{\infty} / \mathcal{C}_{\infty}\right)_{\chi}$. Let $h_{\chi}^{\prime}$ be a divisor of $h_{\chi}$ such that $h_{\chi} / h_{\chi}^{\prime}$ is prime to char $\Lambda_{\infty, \chi}\left(\mathrm{A}_{\infty, \chi}\right)$, and such that any height one prime ideal of $\Lambda_{\infty, \chi}$ which divides $h_{\chi}^{\prime}$ also divides $\operatorname{char}_{\Lambda_{\infty, \chi}}\left(\mathrm{A}_{\infty, \chi}\right)$.

LEMMA 4.8. There is an ideal $\mathcal{I}_{1}$ of $\Lambda_{\infty, \chi}$ of height greater than 1 , and there is $\tilde{h}_{\chi} \in$ $\Lambda_{\infty, \chi}$ prime to $\operatorname{char}_{\Lambda_{\infty, \chi}}\left(\mathrm{A}_{\infty, \chi}\right)$ such that $\mathcal{I}_{1} p^{4 c_{\bar{n}}} \tilde{h}_{\chi} h_{\chi}^{\prime} \subseteq \vartheta_{n, \bar{n}, \chi}\left(\operatorname{Im}\left(\mathcal{C}_{n, \bar{n}, \chi}\right)\right)$ in $Z_{p}[\chi][\Gamma /$ $\left.\Gamma_{n, \bar{n}}\right]$ for all nonnegative integers $\bar{n} \leq n$, where $\operatorname{Im}\left(\mathcal{C}_{n, \bar{n}, \chi}\right)$ is the canonical image of $\mathcal{C}_{n, \bar{n}, \chi}$ in $\mathcal{E}_{n, \bar{n}, \chi}$.

PROOF. The ideal $\mathcal{N}^{\prime}:=\operatorname{Nul}_{\Lambda_{\infty, \chi}}\left(h_{\chi} \cdot\left(\mathcal{E}_{\infty} / \mathcal{C}_{\infty}\right)_{\chi}\right)$ is of height greater than 1 since $h_{\chi} \cdot\left(\mathcal{E}_{\infty} / \mathcal{C}_{\infty}\right)_{\chi}$ is pseudo-null. The ideal $\mathcal{N}^{\prime \prime}:=\operatorname{pr} \cdot \Theta_{\chi}\left(\mathcal{E}_{\infty, \chi}\right)$ is of height greater than 1 since $\operatorname{Cok}\left(\operatorname{pr} \cdot \Theta_{\chi}\right)$ is pseudo-null. We set $\mathcal{I}_{1}:=\mathcal{N}^{\prime} \mathcal{N}^{\prime \prime}$. Then $\mathcal{I}_{1} h_{\chi} \subseteq \operatorname{pr} \cdot \Theta_{\chi}\left(\operatorname{Im}\left(\mathcal{C}_{\infty, \chi}\right)\right)$. For all $i \in \mathcal{I}_{1}$, there is $z_{i} \in \operatorname{Im}\left(\mathcal{C}_{\infty, \chi}\right)$ such that $i h_{\chi}=\operatorname{pr} \cdot \Theta_{\chi}\left(z_{i}\right)$. An easy calculation gives

$$
p^{4 c_{\bar{n}}}\left(\gamma_{\delta^{\prime}}^{\prime}-1\right)^{4}\left(\bar{\gamma}_{\bar{m}_{0}}-1\right)^{8} i h_{\chi}=\vartheta_{n, \bar{n}, \chi}\left(\bar{z}_{i}\right) \quad \text { in } \quad Z_{p}[\chi]\left[\Gamma / \Gamma_{n, \bar{n}}\right],
$$

where $\bar{z}_{i}$ is the image of $z_{i}$ in $\mathcal{E}_{n, \bar{n}, \chi}$. Then we set $\tilde{h}_{\chi}:=\left(\gamma_{\delta^{\prime}}^{\prime}-1\right)^{4}\left(\bar{\gamma}_{\bar{m}_{0}}-1\right)^{8} h_{\chi}\left(h_{\chi}^{\prime}\right)^{-1}$. Remark that $\tilde{h}_{\chi}$ is prime to $\operatorname{char}_{\Lambda_{\infty, \chi}}\left(\mathrm{A}_{\infty, \chi}\right)$ by Remark 2.7 and by definition of $h_{\chi}^{\prime}$. 
5. Euler systems. Let us write $A_{k}$ as a direct product of cyclic $p$-groups,

$$
A_{k}=\left\langle\operatorname{cl}\left(\mathfrak{p}_{1}\right)\right\rangle \times \cdots \times\left\langle\operatorname{cl}\left(\mathfrak{p}_{r}\right)\right\rangle,
$$

where $\mathfrak{p}_{1}, \ldots, \mathfrak{p}_{r}$ are prime ideals of $\mathcal{O}_{k}$, prime to $p$, and $\operatorname{cl}\left(\mathfrak{p}_{i}\right)$ is the class of $\mathfrak{p}_{i}$ in $\operatorname{Cl}\left(\mathcal{O}_{k}\right)$. For any $i \in\{1, \ldots, r\}$, we let $p^{R_{i}}$ be the order of $\left\langle\operatorname{cl}\left(\mathfrak{p}_{i}\right)\right\rangle$, and we choose $\alpha_{i} \in \mathcal{O}_{k}$ such that $\alpha_{i} \mathcal{O}_{k}=\mathfrak{p}_{i}^{p_{i}}$. Let $R:=\sum_{i=1}^{r} R_{i}$ and let $M \neq 1$ be a power of $p$, such that $p^{R}=\#\left(A_{k}\right) \leq M$.

Let $k^{\text {ab }}$ be the abelian closure of $k$ in $C$, and let $F \subset k^{\text {ab }}$ be a finite abelian extension of $k$. We set $\mu_{M}:=\mu_{M}\left(k^{\mathrm{ab}}\right)$. We denote by $\mathcal{L}_{F}$ the set of maximal ideals $\ell$ of $\mathcal{O}_{k}$ such that $\ell$ splits completely in $F\left(\mu_{M}, \sqrt[M]{\alpha_{1}}, \ldots, \sqrt[M]{\alpha_{r}}\right) / k$, and such that $\ell \notin\left\{\mathfrak{p}_{1}, \ldots, \mathfrak{p}_{r}\right\}$. We denote by $\mathcal{S}_{F}$ the set of squarefree ideals of $\mathcal{O}_{k}$ whose prime divisors belongs to $\mathcal{L}_{F}$. As in [10, Lemma 3.1], we define for each $\ell \in \mathcal{L}_{F}$ a cyclic subextension $F(\ell)$ of $\mathrm{H}(\ell) F$, of degree $M$ over $F$, which is totally ramified above $\ell$ and unramified anywhere else. We denote its Galois group by $G_{\ell}$, and we fix a generator $\sigma_{\ell}$ of $G_{\ell}$. For $\mathfrak{m}:=\ell_{1} \cdots \ell_{n}$ an ideal in $\mathcal{S}_{F}$, we define $F(\mathfrak{m}):=$ $F\left(\ell_{1}\right) \cdots F\left(\ell_{n}\right)$, the composite of the fields $F\left(\ell_{i}\right)$. We set $G_{\mathfrak{m}}:=\operatorname{Gal}(F(\mathfrak{m}) / F) \simeq \prod_{\ell \mid \mathfrak{m}} G_{\ell}$.

For any ideal $\mathfrak{m} \neq 0$ of $\mathcal{O}_{k}$, we denote by $\mathcal{S}_{F}(\mathfrak{m})$ the set of ideals in $\mathcal{S}_{F}$ which are prime to $\mathfrak{m}$. We denote by $\mathscr{U}_{F}(\mathfrak{m})$ the set of maps $\varepsilon: \mathcal{S}_{F}(\mathfrak{m}) \rightarrow\left(k^{\mathrm{ab}}\right)^{\times}$satisfying the conditions (a) to (d) below.

(a) $\varepsilon(\mathfrak{a}) \in F(\mathfrak{a})^{\times}$for all $\mathfrak{a} \in \mathcal{S}_{F}(\mathfrak{m})$.

(b) $\varepsilon(\mathfrak{a}) \in \mathcal{O}_{F(\mathfrak{a})}^{\times}$if $\mathfrak{a} \neq(1)$.

(c) $\mathrm{N}_{F(\mathfrak{a} \ell) / F(\mathfrak{a})}(\varepsilon(\mathfrak{a} \ell))=\varepsilon(\mathfrak{a})^{(\ell, F(\mathfrak{a}) / k)-1}$ for all $\mathfrak{a} \in \mathcal{S}_{F}(\mathfrak{m})$ and all $\ell \in \mathcal{L}_{F}$ which is prime to $\mathfrak{m a}$.

(d) $\varepsilon(\mathfrak{a} \ell) \equiv \varepsilon(\mathfrak{a})^{(\mathrm{N}(\ell)-1) / M}$ modulo all prime ideals of $\mathcal{O}_{F(\mathfrak{a} \ell)}$ above $\ell$.

For any ideal $\mathfrak{a} \neq(0)$ of $\mathcal{O}_{k}$, any $\varepsilon \in \mathscr{U}_{F}(\mathfrak{a})$ and any $\mathfrak{m} \in \mathcal{S}_{F}(\mathfrak{a})$, one can construct (in a canonical way) an inhomogeneous 1-cocycle $c: G_{\mathfrak{m}} \rightarrow F(\mathfrak{m})^{\times}$such that $c(\sigma)^{M}=\varepsilon(\mathfrak{m})^{D_{\mathfrak{m}}(\sigma-1)}$ for all $\sigma \in G_{\mathfrak{m}}$, where $D_{\mathfrak{m}}:=\prod_{\ell \mid \mathfrak{m}} \sum_{i=1}^{M-1} i \sigma_{\ell}^{i}$ belongs to $\boldsymbol{Z}\left[G_{\mathfrak{m}}\right]$. Since $\mathrm{H}^{1}\left(G_{\mathfrak{m}}, F(\mathfrak{m})^{\times}\right)=0$, one can find $\beta(\mathfrak{m}) \in F(\mathfrak{m})^{\times}$such that $c(\sigma)=\beta(\mathfrak{m})^{\sigma-1}$ for all $\sigma \in G_{\mathfrak{m}}$ (if $\mathfrak{m}=1$ one takes $\beta(1)=1$ ). We set $\kappa_{\varepsilon}: \mathcal{S}_{F}(\mathfrak{a}) \rightarrow F^{\times} /\left(F^{\times}\right)^{M}$, $\mathfrak{m} \mapsto \varepsilon(\mathfrak{m})^{D_{\mathfrak{m}}} \beta(\mathfrak{m})^{-M}$. This construction is due to Rubin. We refer the readers to [16, Proposition 2.2] for details. Notice that $\kappa_{\varepsilon}(1)=\varepsilon(1)$.

Proposition 5.1 (See [16, Proposition 1.2]). Let $\mathscr{U}:=\bigcup \mathscr{U}_{F}(\mathfrak{m})$, where the union is over all nonzero ideals $\mathfrak{m}$ of $\mathcal{O}_{k}$. Then for any $u \in \Psi_{F}$ (in particular for $u \in C_{F}$ ), there exists $\varepsilon \in \mathscr{U}$ such that $\kappa_{\varepsilon}(1)=u$.

PROOF. For any nonzero ideal $\mathfrak{m} \neq(1)$ of $\mathcal{O}_{k}$, any maximal ideal $\mathfrak{q}$ of $\mathcal{O}_{k}$, and any nonzero ideal $\mathfrak{a}$ of $\mathcal{O}_{k}$ prime to $6 \mathfrak{m q}$, by [13, Corollaire 1.3 , (ii-1)] we have

$$
\begin{aligned}
& \mathrm{N}_{k(\mathfrak{m} \mathfrak{q}) / k(\mathfrak{m})}\left(\psi\left(1 ; \mathfrak{m} \mathfrak{q}, \mathfrak{a}^{-1} \mathfrak{m} \mathfrak{q}\right)\right)^{w_{\mathfrak{m}} / w_{\mathfrak{m} \mathfrak{q}}} \\
& \quad= \begin{cases}\psi\left(1 ; \mathfrak{m}, \mathfrak{a}^{-1} \mathfrak{m}\right)^{1-(\mathfrak{q}, \mathrm{H}(\mathfrak{m}) / k)^{-1}} & \text { if } \mathfrak{q} \nmid \mathfrak{m}, \\
\psi\left(1 ; \mathfrak{m}, \mathfrak{a}^{-1} \mathfrak{m}\right) & \text { if } \mathfrak{q} \mid \mathfrak{m}\end{cases}
\end{aligned}
$$


Moreover, when $\mathfrak{q} \nmid \mathfrak{m}$, by [13, Corollaire 1.3, (v-1)] we have

$$
\psi\left(1 ; \mathfrak{m} \mathfrak{q}, \mathfrak{a}^{-1} \mathfrak{m} \mathfrak{q}\right) \equiv \psi\left(1 ; \mathfrak{m}, \mathfrak{a}^{-1} \mathfrak{m}\right)^{(\mathfrak{q}, k(\mathfrak{m}) / k)} \quad \operatorname{modulo}(\mathfrak{q})_{\mathrm{H}(\mathfrak{m} \mathfrak{q})}
$$

From (5.1) we deduce that $\Psi_{F}$ is generated by $\mu(F)$ and by all the norms

$$
\mathrm{N}_{\mathrm{H}(\mathfrak{m}) / \mathrm{H}(\mathfrak{m}) \cap F}\left(\psi\left(1 ; \mathfrak{m}, \mathfrak{a}^{-1} \mathfrak{m}\right)\right),
$$

where $\mathfrak{m} \neq(1)$ is a nonzero ideal of $\mathcal{O}_{k}$ such that $w_{\mathfrak{m}}=1$ and $\mathfrak{a}$ is any nonzero ideal of $\mathcal{O}_{k}$ prime to $6 \mathrm{~m}$. Hence we have to prove the proposition in two particular cases: when $u \in \mu(F)$ and when $u$ is a norm as in (5.3). The case $u \in \mu(F)$ is treated in [16]. If

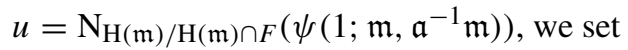

$$
\varepsilon: \mathcal{S}_{F}\left(\mathfrak{m a} w_{F}\right) \rightarrow\left(k^{\mathrm{ab}}\right)^{\times}, \mathfrak{n} \mapsto \mathrm{N}_{\mathrm{H}(\mathfrak{m} \mathfrak{n}) / F(\mathfrak{n}) \cap \mathrm{H}(\mathfrak{m} \mathfrak{n})}\left(\psi\left(1 ; \mathfrak{m n}, \mathfrak{a}^{-1} \mathfrak{m n}\right)\right)^{\prod_{\ell \mid \mathfrak{n}} f_{\ell, \mathfrak{n}}},
$$

where $w_{F}:=\# \mu(F)$ and where for any $\ell \mid \mathfrak{n}, f_{\ell, \mathfrak{n}}$ is the automorphism of $F(\mathfrak{n})$ which is the identity on $F(\ell)$ and the Frobenius of $\ell$ in $F\left(\mathfrak{n} \ell^{-1}\right) / k$. This is well defined since $\ell$ splits completely in $F / k$. The condition (a) is obviously verified, and (b) is implied by (4.1) and (4.2). Conditions (c) and (d) respectively follow from (5.1) and (5.2).

For $\ell \in \mathcal{L}_{F}$, we let $\mathcal{I}_{F, \ell}:=\bigoplus_{\lambda \mid \ell} \boldsymbol{Z} \lambda$ be the free $\boldsymbol{Z}$-module generated by the prime ideals of $\mathcal{O}_{F}$ lying above $\ell$. For any $x \in F^{\times}$, we denote by $(x)_{\ell} \in \mathcal{I}_{F, \ell}$ and $[x]_{\ell} \in \mathcal{I}_{F, \ell} / M \mathcal{I}_{F, \ell}$ the projections of the fractional ideal $(x):=x \mathcal{O}_{F}$. Remark that we have an isomorphism

$$
\left(\mathcal{O}_{F(\ell)} / \ell^{\prime}\right)^{\times} /\left(\left(\mathcal{O}_{F(\ell)} / \ell^{\prime}\right)^{\times}\right)^{M} \simeq\left(\mathcal{O}_{F} / \ell \mathcal{O}_{F}\right)^{\times} /\left(\left(\mathcal{O}_{F} / \ell \mathcal{O}_{F}\right)^{\times}\right)^{M},
$$

where $\ell^{\prime}$ is a product of the prime ideals of $\mathcal{O}_{F(\ell)}$ above $\ell$. For any $z \in F(\ell)^{\times}$, one verifies that the image of $z^{1-\sigma_{\ell}}$ in $\left(\mathcal{O}_{F(\ell)} / \ell^{\prime}\right)^{\times}$is annihilated by $M$. Thus using (5.4) we can consider the map

$$
\theta_{\ell}: F(\ell)^{\times} \longrightarrow\left(\mathcal{O}_{F} / \ell \mathcal{O}_{F}\right)^{\times} /\left(\left(\mathcal{O}_{F} / \ell \mathcal{O}_{F}\right)^{\times}\right)^{M},
$$

which associates to $z$ the sum $\bigoplus_{\lambda \mid \ell} z_{\lambda}$ such that the image of $z^{1-\sigma_{\ell}}$ in $\left(\mathcal{O}_{F} / \lambda\right)^{\times}$is equal to $\left(z_{\lambda}\right)^{(\mathrm{N}(\ell)-1) / M}$. By [16, Proposition 2.3], there exists a unique $\mathrm{Gal}(F / k)$-equivariant isomorphism

$$
\varphi_{\ell}:\left(\mathcal{O}_{F} / \ell \mathcal{O}_{F}\right)^{\times} /\left(\left(\mathcal{O}_{F} / \ell \mathcal{O}_{F}\right)^{\times}\right)^{M} \rightarrow \mathcal{I}_{F, \ell} / M \mathcal{I}_{F, \ell}
$$

satisfying the relation $\left(\varphi_{\ell} \circ \theta_{\ell}\right)(x)=\left[\mathrm{N}_{F(\ell) / F}(x)\right]_{\ell}$ for $x \in F(\ell)^{\times}$. For $x \in F^{\times}$, we can choose $y \in F(\ell)^{\times}$such that $x y^{M}$ is a unit at the prime ideals of $\mathcal{O}_{F(\ell)}$ above $\ell$. We denote by $\left\{x y^{M}\right\}$ the class of $x y^{M}$ in $\left(\mathcal{O}_{F} / \ell \mathcal{O}_{F}\right)^{\times} /\left(\left(\mathcal{O}_{F} / \ell \mathcal{O}_{F}\right)^{\times}\right)^{M}$ under (5.4). Then we set $\varphi_{\ell}(x):=$ $\varphi_{\ell}\left(\left\{x y^{M}\right\}\right)$, which does not depend on the choice of $y$.

By [16, Proposition 2.4], for any ideal $\mathfrak{a} \neq(0)$ of $\mathcal{O}_{k}$, any $\varepsilon \in \mathscr{U}_{F}(\mathfrak{a})$, any $\mathfrak{m} \in \mathcal{S}_{F}(\mathfrak{a})$, and any $\ell \in \mathcal{L}_{F}$, we have

$$
\left[\kappa_{\varepsilon}(\mathfrak{m})\right]_{\ell}= \begin{cases}0 & \text { if } \ell \nmid \mathfrak{m} \\ \varphi_{\ell}\left(\kappa_{\varepsilon}\left(\mathfrak{m} \ell^{-1}\right)\right) & \text { if } \ell \mid \mathfrak{m} .\end{cases}
$$

For any extension $L \subseteq F$ of $k$ and any maximal ideal $\mathfrak{q}$ of $\mathcal{O}_{L}$, we denote by $v_{\mathfrak{q}}$ the normalized valuation at $\mathfrak{q}$, and by $\bar{v}_{\mathfrak{q}}: L^{\times} /\left(L^{\times}\right)^{M} \rightarrow \boldsymbol{Z} / M \boldsymbol{Z}$ the map defined from $v_{\mathfrak{q}}$ by taking the quotient. 
The following theorem is a classical step in the Euler system machinery. The first versions are due to Rubin (see [16, Theorem 3.1]), and to Greither for abelian extensions over $\boldsymbol{Q}$ (see [5, Theorem 3.7]). We took our inspiration in [1, Theorem 3.4].

THEOREM 5.2. Let $\mathfrak{f}$ be the conductor of $F / k$, and set $c:=v_{\mathfrak{p}}(\mathfrak{f})$ and $G_{F}:=$ $\operatorname{Gal}(F / k)$. Assume that we are given an ideal class $\mathfrak{c} \in A_{F}$, a finite $G_{F}$-submodule $W$ of $F^{\times} /\left(F^{\times}\right)^{M}$, and a $G_{F}$-morphism $\Psi: W \rightarrow Z / M Z\left[G_{F}\right]$. Suppose that, for any prime ideal $\mathfrak{q}$ of $F$ which is above $\mathfrak{p}_{i}$ for some $i, \mathfrak{q}$ is unramified in $F / k$ and that $\bar{v}_{\mathfrak{q}}(w)=0$ for any $w \in W$. If $m$ is a positive integer which is divisible by $p^{2 c+1}$, then there are infinitely many maximal ideals $\lambda$ of $\mathcal{O}_{F}$ such that:

(i) $\operatorname{cl}_{p}(\lambda)=\mathfrak{c}^{m}$, where $\mathrm{cl}_{p}(\lambda)$ is the ideal class of $\lambda$ in $A_{F}$,

(ii) $\ell:=\lambda \cap \mathcal{O}_{k}$ belongs to $\mathcal{L}_{F}$,

(iii) for all $w \in W,[w]_{\ell}=0$,

(iv) there exists $u \in(\boldsymbol{Z} / M \boldsymbol{Z})^{\times}$, such that for all $w \in W, \varphi_{\ell}(w)=u p^{2 c+R+1} \Psi(w) \lambda$.

Proof. Let $H_{F}$ be the Hilbert $p$-class field of $F$. Let

$$
F_{i}:= \begin{cases}F\left(\mu_{M}\right) & \text { if } i=0, \\ F_{i-1}\left(\sqrt[M]{\alpha_{i}}\right) & \text { if } 1 \leq i \leq r .\end{cases}
$$

Exactly as in [1, proof of Theorem 3.4], one can prove the following claims.

Claim (A) $\left[H_{F} \cap F\left(\mu_{p^{\infty}}\right): F\right] \leq p^{c}$.

Claim (B) $\operatorname{Gal}\left(H_{F} \cap F_{r}(\sqrt[M]{W}) / F\right)$ is annihilated by $p^{2 c+1}$.

As in [16, Lemma 2.5], one verifies that the canonical map $F^{\times} /\left(F^{\times}\right)^{M} \rightarrow F_{0}^{\times} /\left(F_{0}^{\times}\right)^{M}$ is injective. It implies the following claim.

Claim (C) The canonical map $\mathfrak{K}: \operatorname{Gal}\left(F_{0}(\sqrt[M]{W}) / F_{0}\right) \rightarrow \operatorname{Hom}\left(W, \mu_{M}\right)$ from Kummer theory is an isomorphism.

Let us remark that $F_{i-1}(\sqrt[M]{W}) / F_{i-1}$ is unramified at all the places above $\mathfrak{p}_{i}$ by the hypothesis $\bar{v}_{\mathfrak{q}}(w)=0$ for all $w \in W$ and all $\mathfrak{q} \mid \mathfrak{p}_{i}$. On the other hand, $\left[F_{i}: F_{i-1}\right]$ divides $M$ and the ramification index of any $\mathfrak{q} \mid \mathfrak{p}_{i}$ in $F_{i} / F_{i-1}$ is at least $M p^{-R_{i}}$. Therefore

$$
p^{R_{i}} \text { annihilates } \operatorname{Gal}\left(F_{i-1}(\sqrt[M]{W}) \cap F_{i} / F_{i-1}\right) .
$$

Let $L_{i}:=F_{0}(\sqrt[M]{W}) \cap F_{i}$. As $L_{i} \cap F_{i-1}=L_{i-1}$ we have

$$
\operatorname{Gal}\left(L_{i} / L_{i-1}\right) \simeq \operatorname{Gal}\left(L_{i} F_{i-1} / F_{i-1}\right) .
$$

Since $\operatorname{Gal}\left(L_{i} F_{i-1} / F_{i-1}\right)$ is a quotient of $\operatorname{Gal}\left(F_{i-1}(\sqrt[M]{W}) \cap F_{i} / F_{i-1}\right)$, this implies that $p^{R_{i}}$ annihilates $\operatorname{Gal}\left(L_{i} / L_{i-1}\right)$ thanks to (5.6). In particular, we deduce Claim (D) below.

Claim (D) $\operatorname{Gal}\left(F_{0}(\sqrt[M]{W}) \cap F_{r} / F_{0}\right)$ is annihilated by $p^{R}$.

Let $\zeta$ be a primitive $M$-th root of unity, and $\iota: Z / M Z\left[G_{F}\right] \rightarrow \mu_{M}$ be the morphism such that $\iota(\sigma)=0$ for $\sigma \in G_{F} \backslash\{1\}$ and $\iota(1)=\zeta$. Combining Claim (C) and Claim (D), one may find $\alpha \in \operatorname{Gal}\left(F_{r}(\sqrt[M]{W}) / F_{0}\right)$ such that

$$
\alpha_{\mid F_{r}}=1 \quad \text { and } \quad \mathfrak{K}\left(\alpha_{\mid F_{0}}(\sqrt[M]{W})=(\iota \circ \Psi)^{p^{R}} .\right.
$$


From Claim (B), we may choose $\beta \in \operatorname{Gal}\left(H_{F} F_{r}(\sqrt[M]{W}) / F\right)$ such that

$$
\beta_{\mid F_{r}(\sqrt[M]{W})}=\alpha^{p^{2 c+1}} \quad \text { and } \quad \beta_{\mid H_{F}}=\left(\mathfrak{c}, H_{F} / F\right)^{m} \quad \text { (Artin symbol) } .
$$

Now, from (5.8) we see that $\beta \in \operatorname{Gal}\left(H_{F} F_{r}(\sqrt[M]{W}) / F_{r}\right)$.

By the Čebotarev density theorem, we can find infinitely many primes $\lambda$ in $\mathcal{O}_{F}$, of absolute degree 1, prime to $\prod_{i=1}^{r} \mathfrak{p}_{i}$, such that $\lambda \cap \mathcal{O}_{k}$ is unramified in $H_{F} F_{r}(\sqrt[M]{W}) / k$, and such that the conjugacy class of $\beta$ in $\operatorname{Gal}\left(H_{F} F_{r}(\sqrt[M]{W}) / F\right)$ is the Frobenius of $\lambda$. Then the condition (i) of Theorem 5.2 holds as a consequence of the general properties of the Frobenius. The condition (ii) is also satisfied since $\beta$ is the identity on $F_{r}$. Let $w \in W$. Then for any prime $\lambda^{\prime}$ of $\mathcal{O}_{F_{0}(\sqrt[M]{W})}$ above $\lambda$, we have $\bar{v}_{\lambda}(w)=\bar{v}_{\lambda^{\prime}}(w)=M \bar{v}_{\lambda^{\prime}}(\sqrt[M]{w})=0$, and the condition (iii) follows. Assertion (iv) is proved as in the proof of [16, Theorem 8.1,(iii)].

For any $\boldsymbol{Z}_{p}[G]$-module $N$ and any $z \in N$, we denote by $z_{\chi}$ the canonical image of $z$ in $N_{\chi}$.

LEMMA 5.3. Let $F$ be a finite abelian extension of $k$. Let $\mathcal{G}$ be a subgroup of $G_{F}:=$ $\operatorname{Gal}(F / k)$, and let $\chi$ be an irreducible $\boldsymbol{C}_{p}$-character of $\mathcal{G}$. Let $\ell_{1}, \ldots, \ell_{i} \in \mathcal{L}_{F}$, and for each $j=1, \ldots, i$, let $\lambda_{j}$ be a prime of $\mathcal{O}_{F}$ above $\ell_{j}$, and let $\mathrm{cl}_{p}\left(\lambda_{j}\right)$ be the image of $\lambda_{j}$ in $\mathrm{A}_{F}$. Let $x \in F^{\times}$be such that $v_{\mathfrak{q}}(x) \in M Z$ for any prime $\mathfrak{q}$ of $\mathcal{O}_{F}$ which is prime to $\ell_{1} \cdots \ell_{i} \mathcal{O}_{F}$.

Let $W$ be the $Z_{p}\left[G_{F}\right]$-span of the image $x_{M}$ of $x$ in $F^{\times} /\left(F^{\times}\right)^{M}$, and let $L$ be the $Z_{p}\left[G_{F}\right]$-submodule of $A_{F}$ generated by $\mathrm{cl}_{p}\left(\lambda_{1}\right), \ldots, \mathrm{cl}_{p}\left(\lambda_{i-1}\right)$. Assume that there are $Z, g, \eta, \eta^{\prime} \in Z_{p}\left[G_{F}\right]$ such that

(i) $Z . \operatorname{Nul}_{Z_{p}\left[G_{F}\right]_{\chi}}\left(\left[\mathrm{cl}_{p}\left(\lambda_{i}\right)\right]_{L, \chi}\right) \subseteq g \eta^{\prime} \boldsymbol{Z}_{p}\left[G_{F}\right]_{\chi}$, where $\operatorname{Nul}_{Z_{p}\left[G_{F}\right]_{\chi}}\left(\left[\operatorname{cl}_{p}\left(\lambda_{i}\right)\right]_{L, \chi}\right)$ is the annihilator of the image $\left[\mathrm{cl}_{p}\left(\lambda_{i}\right)\right]_{L, \chi}$ of $\mathrm{cl}_{p}\left(\lambda_{i}\right)$ in $\left(A_{F} / L\right)_{\chi}$,

(ii) $\eta^{\prime} \boldsymbol{Z}_{p}\left[G_{F}\right]_{\chi} / g \eta^{\prime} \boldsymbol{Z}_{p}\left[G_{F}\right]_{\chi}$ is finite,

(iii) $\#\left(\eta\left(\left(\mathcal{I}_{F, \ell_{i}} / M \mathcal{I}_{F, \ell_{i}}\right) / W^{\prime}\right)_{\chi}\right) \#\left(A_{F, \chi}\right) \leq M$, where $W^{\prime}$ is the image of $W$ in $\mathcal{I}_{F, \ell_{i}} /$ $M \mathcal{I}_{F, \ell_{i}}$ through $w \mapsto[w]_{\ell_{i}}$.

Then, there exists a morphism of $\boldsymbol{Z}_{p}\left[G_{F}\right]$-modules $\Psi: W_{\chi} \rightarrow(\boldsymbol{Z} / M \boldsymbol{Z})\left[G_{F}\right]_{\chi}$ such that

$$
g \Psi\left(x_{M, \chi}\right) \lambda_{i, \chi}=Z \eta[x]_{\ell_{i}, \chi} .
$$

PROOF. The proof is similar to that of [5, Lemma 3.12], up to the point that we have to take into account the extra variable $\eta^{\prime}$.

6. The divisibility obtained by Euler systems. This section is devoted to the proof of Proposition 6.1 below.

PROPOSITION 6.1. We have $\operatorname{char}_{\Lambda_{\infty, \chi}}\left(\mathrm{A}_{\infty, \chi}\right) \mid \operatorname{char}_{\Lambda_{\infty, \chi}}\left(\mathcal{E}_{\infty} / \mathcal{C}_{\infty}\right)_{\chi}$.

Let $\mathcal{X}$ be the set of irreducible $\boldsymbol{C}_{p}$-characters $\xi$ of $\Gamma$ such that $\operatorname{Im}(\xi)$ is finite. For all $f \in \Lambda_{\infty, \chi}$, we set

$$
\mathcal{X}(\chi, f):=\{\xi \in \mathcal{X} / \xi(f)=0\} \quad \text { and } \quad \mathcal{J}(\chi, f):=\bigcap_{\xi \in \mathcal{X}(\chi, f)} \operatorname{Ker}(\xi),
$$

where all $\xi \in \mathcal{X}$ are extended to $\Lambda_{\infty, \chi}$ by linearity. 
REMARK 6.2. As in [16, Proposition 7.11 (ii)], one can deduce from Remark 2.7 that $\mathcal{J}(\chi, f)$ is of height greater than 1 whenever $f$ divides $\operatorname{char}_{\Lambda_{\infty, \chi}}\left(\mathrm{A}_{\infty, \chi}\right)$.

Since $\mathcal{J}:=\mathcal{J}\left(\chi, \operatorname{char}_{\Lambda_{\infty, \chi}}\left(\mathrm{A}_{\infty, \chi}\right)\right)$ and $\mathcal{I}_{0} \mathcal{I}_{1}$ are of height greater than 1 , we can choose $\eta^{\prime} \in \mathcal{J}$ and $I \in \mathcal{I}_{0} \mathcal{I}_{1}$ both prime to $\operatorname{char}_{\Lambda_{\infty, \chi}}\left(\mathrm{A}_{\infty, \chi}\right)$. Let $\bar{n} \leq n$ be two nonnegative integers. Let $c_{\bar{n}}^{\prime}$ be the valuation at $\overline{\mathfrak{p}}$ of the conductor of $K_{n, \bar{n}}$ (which does not depend on $n$ ).

For all $i \in \boldsymbol{Z}$, we set

$$
\Delta_{i}:=[K: k]^{i-1} p^{\left(2 c_{\bar{n}}^{\prime}+R+1\right)(2 i-3)+4 c_{\bar{n}}} .
$$

Since $\mathcal{J} \subseteq \mathcal{J}\left(\chi, h_{\chi}^{\prime}\right)$, the group $\eta^{\prime} \boldsymbol{Z}_{p}\left[G_{n, \bar{n}}\right]_{\chi} / \Delta_{s+1} h_{\chi}^{\prime} \eta^{\prime} \boldsymbol{Z}_{p}\left[G_{n, \bar{n}}\right]_{\chi}$ is finite. Let $M \neq 1$ be a power of $p$ such that

$$
\# \mathrm{~A}_{k} \# \mathrm{~A}_{n, \bar{n}, \chi} \#\left(\eta^{\prime} \boldsymbol{Z}_{p}\left[G_{n, \bar{n}}\right]_{\chi} / \Delta_{s+1} h_{\chi}^{\prime} \eta^{\prime} \boldsymbol{Z}_{p}\left[G_{n, \bar{n}}\right]_{\chi}\right) \leq M .
$$

For all maximal ideal $\lambda$ of $\mathcal{O}_{K_{n, \bar{n}}}$ such that $\ell:=\lambda \cap \mathcal{O}_{k} \in \mathcal{L}_{K_{n, \bar{n}}}$, we denote by $\omega_{\lambda}$ and $\bar{\omega}_{\lambda}$ the maps

$$
\omega_{\lambda}: K_{n, \bar{n}}^{\times} \longrightarrow Z_{p}\left[G_{n, \bar{n}}\right] \text { such that } \omega_{\lambda}(x) \lambda=(x)_{\ell},
$$

and

$$
\bar{\omega}_{\lambda}: K_{n, \bar{n}}^{\times} /\left(K_{n, \bar{n}}^{\times}\right)^{M} \longrightarrow(\boldsymbol{Z} / M \boldsymbol{Z})\left[G_{n, \bar{n}}\right] \quad \text { such that } \quad \bar{\omega}_{\lambda}\left(x_{M}\right) \lambda=[x]_{\ell} .
$$

We know by Lemma 2.10 that for every $j \in\{1, \ldots, s\}$, there is a class $\mathfrak{c}_{j} \in A_{n, \bar{n}}$ such that

$$
\tau_{n, \bar{n}, \chi}\left(\mathfrak{c}_{j, \chi}\right)=(0, \ldots, 0, \tilde{I}, 0, \ldots, 0),
$$

where $\tilde{I}:=I\left(\gamma_{\delta^{\prime}}^{\prime}-1\right)^{2}\left(\bar{\gamma}_{\bar{\delta}}-1\right)^{2}(\bar{\gamma}-1)$ is at the $j$-th place. We also choose arbitrarily one more class $\mathfrak{c}_{s+1} \in A_{n, \bar{n}}$. By Lemma 4.8 , there is $\xi \in C_{n, \bar{n}}$ such that

$$
\vartheta_{n, \bar{n}, \chi}\left(\xi^{\prime}\right)=I \tilde{h}_{\chi} p^{4 c_{\bar{n}}} h_{\chi}^{\prime} \quad \text { in } \quad(\boldsymbol{Z} / \boldsymbol{M Z})\left[G_{n, \bar{n}}\right]_{\chi},
$$

where $\xi^{\prime}$ is the image of $\xi$ in $\operatorname{Im}\left(\mathcal{C}_{n, \bar{n}, \chi}\right)$. By Remark 5.1, we can fix an ideal $\mathfrak{m}$ of $\mathcal{O}_{k}$ and $\varepsilon \in \mathscr{U}_{K_{n, \bar{n}}}(\mathfrak{m})$ such that $\kappa_{\varepsilon}(1)=\xi$. We assume that all the $\mathfrak{p}_{i}$ are unramified in $K_{\infty} / k$ and prime to $(p)$ in order to apply Theorem 5.2.

The main step is to define recursively maximal ideals $\lambda_{1}, \ldots, \lambda_{s+1}$ of $\mathcal{O}_{K_{n, \bar{n}}}$ and ideals $\mathfrak{a}_{1}, \ldots, \mathfrak{a}_{s+1}$ of $\mathcal{O}_{k}$ such that

(a) $\ell_{i}:=\lambda_{i} \cap \mathcal{O}_{k}$ belongs to $\mathcal{L}_{K_{n, \bar{n}}}$ for all $i=1, \ldots, s+1$,

(b) $\operatorname{cl}_{p}\left(\lambda_{i}\right)=\mathfrak{c}_{i}^{p^{2 c_{n}^{\prime}+R+1}}$ for all $i=1, \ldots, s+1$,

(c) $\mathfrak{a}_{i}:=\ell_{1} \cdots \ell_{i}$,

(d) $\bar{\omega}_{\lambda_{1}}\left(\kappa_{\varepsilon}\left(\ell_{1}\right)\right)_{\chi}=u_{1} p^{4 c_{\bar{n}}+2 c_{\bar{n}}^{\prime}+R+1}[K: k] I \tilde{h}_{\chi} h_{\chi}^{\prime}$ in $(\boldsymbol{Z} / M \boldsymbol{Z})\left[G_{n, \bar{n}}\right]_{\chi}$, for some $u_{1} \in(\boldsymbol{Z} / M \boldsymbol{Z})^{\times}$,

(e) for every $i \in\{2, \ldots, s+1\}$ there is $u_{i} \in(\boldsymbol{Z} / M \boldsymbol{Z})^{\times}$such that $P_{i-1} \bar{\omega}_{\lambda_{i}}\left(\kappa_{\varepsilon}\left(\mathfrak{a}_{i}\right)\right)_{\chi}=u_{i} p^{4 c_{\bar{n}}^{\prime}+2 R+2}[K: k]\left(\eta^{\prime}\right)^{2^{i-1}} \tilde{I}^{2^{i-2}}\left(I \tilde{h}_{\chi}\right)^{2^{i-2}} \bar{\omega}_{\lambda_{i-1}}\left(\kappa_{\varepsilon}\left(\mathfrak{a}_{i-1}\right)\right)_{\chi}$.

We define a morphism of $\boldsymbol{Z}_{p}\left[G_{n, \bar{n}}\right]$-modules

$$
\varpi: \boldsymbol{Z}_{p}[\chi]\left[\Gamma / \Gamma_{n, \bar{n}}\right] \rightarrow \boldsymbol{Z}_{p}\left[G_{n, \bar{n}}\right], \chi(g) v \mapsto[K: k] e_{\psi} g v
$$


for all $(g, v) \in G \times\left(\Gamma / \Gamma_{n, \bar{n}}\right)$. Let us consider the map $\varpi \circ \vartheta_{n, \bar{n}, \chi} \circ \eta: \mathcal{O}_{K_{n, \bar{n}}}^{\times} \rightarrow \boldsymbol{Z}_{p}\left[G_{n, \bar{n}}\right]$, where $\eta: \mathcal{O}_{K_{n, \bar{n}}}^{\times} \rightarrow \mathcal{E}_{n, \bar{n}, \chi}$ is the natural map. Further, by taking the quotients we obtain a map

$$
\Psi_{1}: \mathcal{O}_{K_{n, \bar{n}}}^{\times} /\left(\mathcal{O}_{K_{n, \bar{n}}}^{\times}\right)^{M} \longrightarrow(\boldsymbol{Z} / M \boldsymbol{Z})\left[G_{n, \bar{n}}\right] .
$$

We apply Theorem 5.2 to the data

$$
F:=K_{n, \bar{n}}, \quad m:=p^{2 c_{\bar{n}}^{\prime}+R+1}, \quad W:=W_{1}, \quad \Psi:=\Psi_{1}, \quad \text { and } \quad \mathfrak{c}:=\mathfrak{c}_{1},
$$

where $W_{1}$ is the $Z_{p}\left[G_{n, \bar{n}}\right]$-span of the image $\xi_{M}$ of $\xi$ in $F^{\times} /\left(F^{\times}\right)^{M}$. We obtain a maximal ideal $\lambda_{1}$ of $\mathcal{O}_{K_{n, \bar{n}}}$ such that $\operatorname{cl}_{p}\left(\lambda_{1}\right)=\mathfrak{c}_{1}^{p^{2 c_{n}^{\prime}+R+1}}$ and such that the ideal $\ell_{1}:=\lambda_{1} \cap \mathcal{O}_{k}$ belongs to $\mathcal{L}_{K_{n, \bar{n}}}$. Moreover, for all $w \in W_{1}$, we have $[w]_{\ell_{1}}=0$ and there is $u_{1} \in(\boldsymbol{Z} / M \boldsymbol{Z})^{\times}$such that for all $w \in W_{1}$, we have

$$
\varphi_{\ell_{1}}(w)=u_{1} p^{2 c_{\bar{n}}^{\prime}+R+1} \Psi_{1}(w) \lambda_{1} .
$$

We denote by $\bar{\vartheta}_{n, \bar{n}, \chi}: \mathcal{E}_{n, \bar{n}, \chi} \rightarrow(\boldsymbol{Z} / M \boldsymbol{Z})\left[G_{n, \bar{n}}\right]_{\chi}$ and $\bar{\varpi}:(\boldsymbol{Z} / M \boldsymbol{Z})\left[G_{n, \bar{n}}\right]_{\chi} \rightarrow$ $(\boldsymbol{Z} / M \boldsymbol{Z})\left[G_{n, \bar{n}}\right]$ the morphisms obtained from $\vartheta_{n, \bar{n}, \chi}$ and $\bar{\varpi}$ respectively, by taking the quotients. By (5.5), we deduce

$$
\left[\kappa_{\varepsilon}\left(\ell_{1}\right)\right]_{\ell_{1}}=\varphi_{\ell_{1}}(\xi)=u_{1} p^{2 c_{\bar{n}}^{\prime}+R+1} \Psi_{1}\left(\xi_{M}\right) \lambda_{1}=u_{1} p^{2 c_{\bar{n}}^{\prime}+R+1} \bar{\varpi} \circ \bar{\vartheta}_{n, \bar{n}, \chi}\left(\xi^{\prime}\right) \lambda_{1}
$$

in $\mathcal{I}_{K_{n, \bar{n}}, \ell_{1}} / M \mathcal{I}_{K_{n, \bar{n}}, \ell_{1}}$. From (6.4) and (6.2), we deduce that in $(\boldsymbol{Z} / \boldsymbol{M Z})\left[G_{n, \bar{n}}\right]_{\chi}$ we have

$$
\begin{aligned}
\bar{\omega}_{\lambda_{1}}\left(\kappa_{\varepsilon}\left(\ell_{1}\right)\right)_{\chi} & =u_{1} p^{2 c_{\bar{n}}^{\prime}+R+1}[K: k] \bar{\vartheta}_{n, \bar{n}, \chi}\left(\xi^{\prime}\right) \\
& =u_{1} p^{4 c_{\bar{n}}+2 c_{\bar{n}}^{\prime}+R+1}[K: k] I \tilde{h}_{\chi} h_{\chi}^{\prime},
\end{aligned}
$$

that is to say (d).

Let $i \in\{2, \ldots, s+1\}$, and assume that $\lambda_{1}, \ldots, \lambda_{i-1}$ has been constructed. From (d) and (e) we deduce

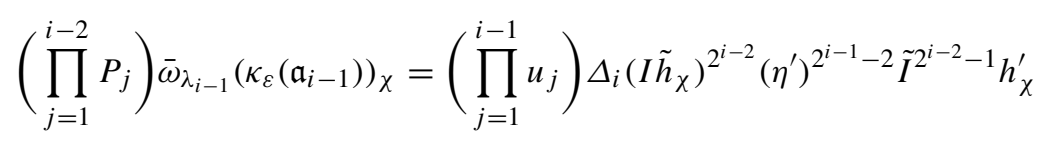

in $(\boldsymbol{Z} / M \boldsymbol{Z})\left[G_{n, \bar{n}}\right]_{\chi}$, with the convention that an empty product is 1 and an empty sum is 0 .

LEMMA 6.3. Let $L_{i}$ be the $\boldsymbol{Z}_{p}\left[G_{n, \bar{n}}\right]$-submodule of $\mathrm{A}_{n, \bar{n}}$ generated by $\mathrm{cl}_{p}\left(\lambda_{1}\right), \ldots$, $\mathrm{cl}_{p}\left(\lambda_{i-2}\right)$, and let $W_{i}$ be the $\boldsymbol{Z}_{p}\left[G_{n, \bar{n}}\right]$-span of the image of $\kappa_{\varepsilon}\left(\mathfrak{a}_{i-1}\right)$ in $K_{n, \bar{n}}^{\times} /\left(K_{n, \bar{n}}^{\times}\right)^{M}$. We set $\eta_{i}:=\left(I \tilde{h}_{\chi}\right)^{2^{i-2}}\left(\eta^{\prime}\right)^{2^{i-1}-1} \tilde{I}^{2^{i-2}-1}, Z_{i}:=\eta^{\prime} \tilde{I} p^{2 c_{\bar{n}}^{\prime}+R+1}$, and we choose $g_{i} \in Z_{p}\left[G_{n, \bar{n}}\right]$ such that the image of $g_{i}$ in $\boldsymbol{Z}_{p}\left[G_{n, \bar{n}}\right]_{\chi}$ is equal to that of $P_{i-1}$. Then

(i) $v_{\mathfrak{q}}\left(\kappa_{\varepsilon}\left(\mathfrak{a}_{i-1}\right)\right) \in M \boldsymbol{Z}$ for any maximal ideal $\mathfrak{q}$ of $\mathcal{O}_{K_{n, \bar{n}}}$ which is prime to $\mathfrak{a}_{i-1}$,

(ii) $Z_{i} \cdot \operatorname{Nul}_{Z_{p}\left[G_{n, \bar{n}}\right]_{\chi}}\left(\left[\operatorname{cl}_{p}\left(\lambda_{i-1}\right)\right]_{L_{i}, \chi}\right) \subseteq g_{i} \eta^{\prime} Z_{p}\left[G_{n, \bar{n}}\right]_{\chi}$, where $\left[\operatorname{cl}_{p}\left(\lambda_{i-1}\right)\right]_{L_{i}, \chi}$ is the image of $\mathrm{cl}_{p}\left(\lambda_{i-1}\right)$ in $\left(\mathrm{A}_{n, \bar{n}} / L_{i}\right)_{\chi}$,

(iii) $\eta^{\prime} \boldsymbol{Z}_{p}\left[G_{n, \bar{n}}\right]_{\chi} / g_{i} \eta^{\prime} \boldsymbol{Z}_{p}\left[G_{n, \bar{n}}\right]_{\chi}$ is finite,

(iv) $\#\left(\eta_{i}\left(\left(\mathcal{I}_{K_{n, \bar{n}}, \ell_{i-1}} / M \mathcal{I}_{K_{n, \bar{n}}, \ell_{i-1}}\right) / W_{i}^{\prime}\right)_{\chi}\right) \#\left(\mathrm{~A}_{n, \bar{n}, \chi}\right) \leq M$, where $W_{i}^{\prime}$ is the image of $W_{i}$ in $\mathcal{I}_{K_{n, \bar{n}}, \ell_{i-1}} / M \mathcal{I}_{K_{n, \bar{n}}, \ell_{i-1}}$ through $w \mapsto[w]_{\ell_{i-1}}$. 
PROOF. (i) is a direct consequence of (5.5). (iii) is due to the fact that $\eta^{\prime} \in \mathcal{J}$ and $\mathcal{J} \subseteq \mathcal{J}\left(\chi, P_{i-1}\right)$. We can define from $\tau_{n, \bar{n}, \chi}$ a morphism of $\boldsymbol{Z}_{p}\left[G_{n, \bar{n}}\right]_{\chi}$-modules

$$
\tau_{n, \bar{n}, \chi}^{\prime}:\left(\mathrm{A}_{n, \bar{n}} / L_{i}\right)_{\chi} \rightarrow \boldsymbol{Z}_{p}\left[G_{n, \bar{n}}\right]_{\chi} / g_{i} \boldsymbol{Z}_{p}\left[G_{n, \bar{n}}\right]_{\chi}
$$

such that the diagram

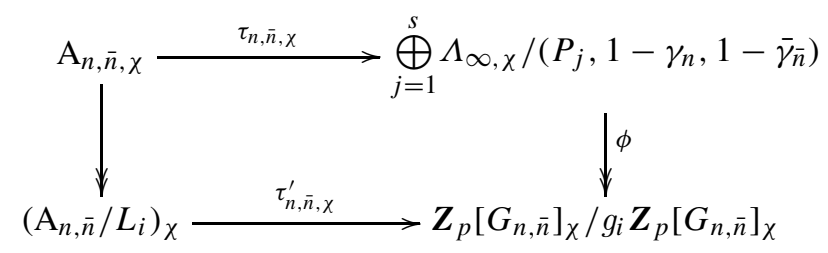

commutes, where $\phi$ is the canonical projection

$\bigoplus_{j=1}^{s} \Lambda_{\infty, \chi} /\left(P_{j}, 1-\gamma_{n}, 1-\bar{\gamma}_{\bar{n}}\right) \longrightarrow \Lambda_{\infty, \chi} /\left(P_{i-1}, 1-\gamma_{n}, 1-\bar{\gamma}_{\bar{n}}\right) \simeq Z_{p}\left[G_{n, \bar{n}}\right]_{\chi} / g_{i} Z_{p}\left[G_{n, \bar{n}}\right]_{\chi}$.

Let $\alpha \in \operatorname{Nul}_{Z_{p}\left[G_{n, \bar{n}}\right]_{\chi}}\left(\left[\operatorname{cl}_{p}\left(\lambda_{i-1}\right)\right]_{L_{i}, \chi}\right)$. Then $\alpha p^{2 c_{\bar{n}}^{\prime}+R+1}\left(\phi \circ \tau_{n, \bar{n}, \chi}\right)\left(\mathfrak{c}_{i-1, \chi}\right)=0$, i.e., $p^{2 c_{\bar{n}}^{\prime}+R+1} \tilde{I} \alpha \in g_{i} \boldsymbol{Z}_{p}\left[G_{n, \bar{n}}\right]_{\chi}$, and (ii) is verified. From (6.6), we see that $\eta_{i}\left(\left(\mathcal{I}_{K_{n, \bar{n}}, \ell_{i-1}} /\right.\right.$ $\left.\left.M \mathcal{I}_{K_{n, \bar{n}}, \ell_{i-1}}\right) / W_{i}^{\prime}\right)_{\chi}$ is a quotient of $\eta^{\prime}\left(\boldsymbol{Z}_{p}\left[G_{n, \bar{n}}\right]_{\chi} / \Delta_{i} h_{\chi}^{\prime} \boldsymbol{Z}_{p}\left[G_{n, \bar{n}}\right]_{\chi}\right)$. The inequality (6.1) then implies (iv).

Let us apply Lemma 5.3 to the material furnished in Lemma 6.3. There is a morphism of $\boldsymbol{Z}_{p}\left[G_{n, \bar{n}}\right]$-modules $\Psi_{i}^{\prime}: W_{i, \chi} \rightarrow(\boldsymbol{Z} / M \boldsymbol{Z})\left[G_{n, \bar{n}}\right]_{\chi}$ such that

$$
g_{i} \Psi_{i}^{\prime}\left(\kappa_{\varepsilon}\left(\mathfrak{a}_{i-1}\right)_{M, \chi}\right) \lambda_{i-1, \chi}=Z_{i} \eta_{i}\left[\kappa_{\varepsilon}\left(\mathfrak{a}_{i-1}\right)\right]_{\ell_{i-1}, \chi} .
$$

We define $\Psi_{i}$ by composing $\varpi \circ \Psi_{i}^{\prime}$ with $W_{i} \rightarrow W_{i, \chi}$. The condition ( $i$ ) of Lemma 6.3 allows us to apply Theorem 5.2 to the data

$$
F:=K_{n, \bar{n}}, \quad m:=p^{2 c_{\bar{n}}^{\prime}+R+1}, \quad \mathfrak{c}:=\mathfrak{c}_{i}, \quad W:=W_{i}, \quad \text { and } \quad \Psi:=\Psi_{i} .
$$

We obtain a maximal ideal $\lambda_{i}$ of $\mathcal{O}_{K_{n, \bar{n}}}$ such that $\operatorname{cl}_{p}\left(\lambda_{i}\right)=\mathfrak{c}_{i}^{p^{2 c_{\bar{n}}^{\prime}+R+1}}$ (condition (b)) and such that $\ell_{i}:=\lambda_{i} \cap \mathcal{O}_{k}$ belongs to $\mathcal{L}_{K_{n, \bar{n}}}$ (condition (a)). Moreover, for all $w \in W_{i}$, we have $[w]_{\ell_{i}}=0$ and there is $u_{i} \in(\boldsymbol{Z} / M \boldsymbol{Z})^{\times}$such that for all $w \in W_{i}$, we have $\varphi_{\ell_{i}}(w)=$ $u_{i} p^{2 c_{\bar{n}}^{\prime}+R+1} \Psi_{i}(w) \lambda_{i}$. By (5.5) we have

$$
\left[\kappa_{\varepsilon}\left(\mathfrak{a}_{i}\right)\right]_{\ell_{i}, \chi}=\varphi_{\ell_{i}}\left(\kappa_{\varepsilon}\left(\mathfrak{a}_{i-1}\right)\right)_{\chi}=u_{i} p^{2 c_{\bar{n}}^{\prime}+R+1} \Psi_{i}\left(\kappa_{\varepsilon}\left(\mathfrak{a}_{i-1}\right)_{M}\right) \lambda_{i, \chi}
$$

in $\left(\mathcal{I}_{K_{n, \bar{n}}, \ell_{i}} / M \mathcal{I}_{K_{n, \bar{n}}, \ell_{i}}\right)_{\chi}$. Then in $(\boldsymbol{Z} / M \boldsymbol{Z})\left[G_{n, \bar{n}}\right]_{\chi}$, by (6.8) and (6.7) we have

$$
\begin{aligned}
P_{i-1} \bar{\omega}_{\lambda_{i}}\left(\kappa_{\varepsilon}\left(\mathfrak{a}_{i}\right)\right)_{\chi} & =u_{i}[K: k] p^{2 c_{\bar{n}}^{\prime}+R+1} g_{i} \Psi_{i}^{\prime}\left(\kappa_{\varepsilon}\left(\mathfrak{a}_{i-1}\right)_{M, \chi}\right) \\
& =u_{i}[K: k] p^{2 c_{\bar{n}}^{\prime}+R+1} Z_{i} \eta_{i} \bar{\omega}_{\lambda_{i-1}}\left(\kappa_{\varepsilon}\left(\mathfrak{a}_{i-1}\right)\right)_{\chi},
\end{aligned}
$$

which demonstrates (e). 
So we can construct recursively the primes $\lambda_{1}, \ldots, \lambda_{s+1}$, and from (d) and (e) we deduce

$$
\left(\prod_{j=1}^{s} P_{j}\right) \bar{\omega}_{\lambda_{s+1}}\left(\kappa_{\varepsilon}\left(\mathfrak{a}_{s+1}\right)\right)_{\chi}=\left(\prod_{j=1}^{s+1} u_{j}\right) \Delta_{s+2}\left(I \tilde{h}_{\chi}\right)^{2^{s}}\left(\eta^{\prime}\right)^{2^{s+1}-2} \tilde{I}^{2^{s}-1} h_{\chi}^{\prime}
$$

in $(\boldsymbol{Z} / M \boldsymbol{Z})\left[G_{n, \bar{n}}\right]_{\chi}$. By letting $M$ and $n$ vary, this implies that

$$
\prod_{j=1}^{s} P_{j} \quad \text { divides } \quad \Delta_{s+2}\left(I \tilde{h}_{\chi}\right)^{2^{s}}\left(\eta^{\prime}\right)^{2^{s+1}-2} \tilde{I}^{2^{s}-1} h_{\chi}^{\prime}
$$

in $Z_{p}[\chi]\left[\left[\Upsilon^{\prime}\right]\right]\left[\bar{\Upsilon} / \bar{\Upsilon}_{\bar{n}}\right]$. Let $\left(a_{l}\right)_{l \in N} \in\left(Z_{p}[\chi]\left[\left[\Upsilon^{\prime}\right]\right]\right)^{N}$ be such that $\prod_{j=1}^{s} P_{j}=\sum_{l=0}^{\infty} a_{l} \bar{T}^{l}$, where $\bar{T}:=\bar{\gamma}-1$. By Proposition 2.8, $a_{0}$ is prime to $\mathfrak{u}_{\chi}$ in $\boldsymbol{Z}_{p}[\chi]\left[\left[\Upsilon^{\prime}\right]\right]$. From Lemma 6.4 below, we deduce that $\prod_{j=1}^{s} P_{j}$ divides $\left(I \tilde{h}_{\chi}\right)^{2^{s}}\left(\eta^{\prime}\right)^{2^{s+1}-2} \tilde{I}^{2^{s}-1} h_{\chi}^{\prime}$ in $Z_{p}[\chi]\left[\left[\Upsilon^{\prime}\right]\right]\left[\bar{\Upsilon} \bar{\Upsilon}_{\bar{n}}\right]$. By letting $\bar{n}$ vary, $\prod_{j=1}^{s} P_{j}$ divides $\left(I \tilde{h}_{\chi}\right)^{2^{s}}\left(\eta^{\prime}\right)^{2^{s+1}-2} \tilde{I}^{2^{s}-1} h_{\chi}^{\prime}$ in $\Lambda_{\infty, \chi}$. Since $\prod_{j=1}^{s} P_{j}$ is prime to $\left(I \tilde{h}_{\chi}\right)^{2^{s}}\left(\eta^{\prime}\right)^{2^{s+1}-2} \tilde{I}^{2^{s}-1}$, we deduce Proposition 6.1.

LemMA 6.4. We identify $\Lambda_{\infty, \chi}$ with $\boldsymbol{Z}_{p}[\chi]\left[\left[\Upsilon^{\prime}\right]\right][[\bar{T}]]$. Let $a:=\sum_{l=0}^{\infty} a_{l} \bar{T}^{l}$ and $b$ be two elements of $\Lambda_{\infty, \chi}$ such that $a_{0}$ is prime to $\mathfrak{u}_{\chi}$. Let $\bar{n} \in N$. We assume that there is $m \in N$ such that a divides $p^{m} b$ in $\boldsymbol{Z}_{p}[\chi]\left[\left[\Upsilon^{\prime}\right]\right]\left[\bar{\Upsilon} / \bar{\Upsilon}_{\bar{n}}\right]$. Then a divides $b$ in $\boldsymbol{Z}_{p}[\chi]\left[\left[\Upsilon^{\prime}\right]\right]\left[\bar{\Upsilon} / \bar{\Upsilon}_{\bar{n}}\right]$.

PROOF. We can assume that $m \in N$ is minimal such that $a$ divides $p^{m} b$ in $Z_{p}[\chi]\left[\left[\Upsilon^{\prime}\right]\right]$ $\left[\bar{\Upsilon} / \bar{\Upsilon}_{\bar{n}}\right]$. We assume that $m \neq 0$, and then we will find a contradiction to the minimality of $m$. There is an element $\alpha$ of $\Lambda_{\infty, \chi}$ such that $\left(a \alpha-p^{m} b\right) \in\left(1-\bar{\gamma}_{\bar{n}}\right)$. Let $\left(\beta_{l}\right)_{l=0}^{p^{\bar{n}}-1} \in$ $\left(\boldsymbol{Z}_{p}[\chi]\left[\left[\Upsilon^{\prime}\right]\right]\right)^{p^{\bar{n}}}$ be such that $\alpha \equiv \beta$ modulo $\left(1-\bar{\gamma}_{\bar{n}}\right)$, where $\beta:=\sum_{l=0}^{p^{\bar{n}}-1} \beta_{l} \bar{T}^{l}$. For all $s:=\sum_{j=0}^{\infty} s_{l} \bar{T}^{l}$ in $\left(1-\bar{\gamma}_{\bar{n}}\right)$, there is $z:=\sum_{j=0}^{\infty} z_{l} \bar{T}^{l}$ in $\Lambda_{\infty, \chi}$ such that

$$
s=\left(\bar{\gamma}_{\bar{n}}-1\right) z=\sum_{j=1}^{\infty}\left(\sum_{l=1}^{\min \left\{j, p^{\bar{n}}\right\}}\left(\begin{array}{c}
p^{\bar{n}} \\
l
\end{array}\right) z_{j-l}\right) \bar{T}^{j} .
$$

Then for any $j \in\left\{1, \ldots, p^{\bar{n}}-1\right\}$ and any $l \in\{1, \ldots, j\}$, we have $p \mid\left(\begin{array}{c}p^{\bar{n}} \\ l\end{array}\right)$, hence $p \mid s_{j}$. Moreover $s_{0}=0$. Considering $s:=a \beta-p^{m} b$, we obtain

$$
\left\{\begin{array}{l}
a_{0} \beta_{0}=p^{m} b_{0} \\
a_{0} \beta_{j} \equiv-\sum_{l=1}^{j} a_{l} \beta_{j-l} \quad \text { modulo } \quad p \quad \text { for all } j \in\left\{1, \ldots, p^{\bar{n}}-1\right\},
\end{array}\right.
$$

where $b:=\sum_{l=1}^{\infty} b_{l} \bar{T}^{l}$. Since $a_{0}$ is prime to $\mathfrak{u}_{\chi}$, we deduce $p \mid \beta_{i}$ for all $i \in\left\{0, p^{\bar{n}}-1\right\}$. Then $p \mid \beta$, and setting $\tilde{\alpha}:=p^{-1} \beta$ we have $\left(a \tilde{\alpha}-p^{m-1} b\right) \in\left(1-\bar{\gamma}_{\bar{n}}\right)$, which contradicts the minimality of $m$.

7. Proof of Theorem 1.1. We let $G$ act on $\mathcal{Q} \otimes_{Z_{p}} \Lambda_{\Upsilon^{\prime}}$ through $\chi$, and $\Gamma / \bar{\Upsilon} \simeq \Upsilon^{\prime}$ act naturally on $\mathcal{Q} \otimes z_{p} \Lambda_{\Upsilon^{\prime}}$. These two actions commute, so that $\mathcal{Q} \otimes z_{p} \Lambda_{\Upsilon^{\prime}}$ is a $\Lambda_{\infty}$-module. 
LEMMA 7.1. We have

$$
\mathcal{J}_{\infty} \operatorname{char}_{\mathcal{Q} \otimes \mathbf{z}_{p} \Lambda_{\Upsilon^{\prime}}}\left(e_{\chi} \mathcal{Q} \otimes \mathbf{z}_{p} \operatorname{Cok}\left(\pi_{\infty, 0}^{\mathcal{C}}\right)\right)=\mathcal{J}_{\bar{D}} \cdot\left(\mathcal{Q} \otimes \mathbf{z}_{p} \Lambda_{\Upsilon^{\prime}}\right) .
$$

Proof. We denote by $\tilde{\chi}$ the character on $G_{\infty}$ defined by $\chi$. If $\tilde{\chi}$ is not trivial on $\bar{J}$, then

$$
\mathcal{J}_{\infty} \cdot\left(\mathcal{Q} \otimes \mathbf{Z}_{p} \Lambda_{\Upsilon^{\prime}}\right)=\mathcal{J}_{\bar{D}} \cdot\left(\mathcal{Q} \otimes \mathbf{z}_{p} \Lambda_{\Upsilon^{\prime}}\right)=\mathcal{Q} \otimes \mathbf{z}_{p} \Lambda_{\Upsilon^{\prime}}
$$

If $\tilde{\chi}$ is trivial on $\bar{J}$ and $\chi \neq 1$, then $\mathcal{J}_{\infty} \cdot\left(\mathcal{Q} \otimes \boldsymbol{Z}_{p} \Lambda_{\Upsilon^{\prime}}\right)=\mathcal{Q} \otimes \boldsymbol{Z}_{p} \Lambda_{\Upsilon^{\prime}}$ and $\mathcal{J}_{\bar{D}} \cdot\left(\mathcal{Q} \otimes \boldsymbol{Z}_{p} \Lambda_{\Upsilon^{\prime}}\right)$ is generated by $1-\chi(g) v$, where $(g, v) \in G \times \Upsilon^{\prime}$ is such that $\bar{D} /(\bar{J}(\bar{D} \cap \bar{\Upsilon}))$ is topologically generated by $g v$. If $\chi=1$, then $\mathcal{J}_{\infty} \cdot\left(\mathcal{Q} \otimes \mathbf{z}_{p} \Lambda_{\Upsilon^{\prime}}\right)$ is generated by $1-\gamma^{\prime}$, and $\mathcal{J}_{\bar{D}} \cdot\left(\mathcal{Q} \otimes \mathbf{z}_{p} \Lambda_{\Upsilon^{\prime}}\right)$ is generated by $1-\left(\gamma^{\prime}\right)^{p^{n}}$, where $n \in N$ is such that the image of $\bar{D}$ through the projection $G_{\infty} \rightarrow \Upsilon^{\prime}$ is $\Upsilon_{n}^{\prime}$. In all cases, $\mathcal{J}_{\infty} \cdot\left(\mathcal{Q} \otimes z_{p} \Lambda_{\Upsilon^{\prime}}\right)$ and $\mathcal{J}_{\bar{D}} \cdot\left(\mathcal{Q} \otimes z_{p} \Lambda_{\Upsilon^{\prime}}\right)$ are principal ideals and then Lemma 7.1 follows from Proposition 4.4.

\section{LEMMA 7.2. We have}

$$
\begin{aligned}
& \mathcal{J}_{\infty} \operatorname{char}_{\mathcal{Q} \otimes \mathbf{z}_{p} \Lambda_{\Gamma}}\left(e_{\chi} \mathcal{Q} \otimes \mathbf{z}_{p} \mathrm{~A}_{\infty}\right) \operatorname{char}_{\mathcal{Q} \otimes \mathbf{z}_{p} \Lambda_{\gamma^{\prime}}}\left(e_{\chi} \mathcal{Q} \otimes \mathbf{z}_{p} \operatorname{Cok}\left(\pi_{\infty, 0}^{\mathcal{E}}\right)\right) \\
& =\mathcal{J}_{\bar{D}} \operatorname{char}_{\mathcal{Q} \otimes z_{p} \Lambda_{\gamma^{\prime}}}\left(e_{\chi} \mathcal{Q} \otimes z_{p} A_{\infty, 0}\right)
\end{aligned}
$$

in $\mathcal{Q} \otimes \mathrm{Z}_{p} \Lambda_{\Upsilon^{\prime}}$

PROOF. We set $c(N):=\operatorname{char}_{\mathcal{Q} \otimes \mathbf{z}_{p} \Lambda_{\Gamma}}\left(e_{\chi} \mathcal{Q} \otimes \mathbf{z}_{p} N\right)$ for any finitely generated torsion $\Lambda_{\infty}$-module $N$. Also, we set $c^{\prime}(P):=\operatorname{char}_{\mathcal{Q} \otimes_{z_{p}} \Lambda_{\gamma^{\prime}}}\left(e_{\chi} \mathcal{Q} \otimes_{Z_{p}} P\right)$ for any finitely generated torsion $\Lambda_{\Upsilon^{\prime}}$-module $P$. Applying the snake lemma to (3.7) and using (3.1) and Theorem 2.4 (iii), we obtain

$$
c^{\prime}\left(\left(\mathrm{A}_{\infty}\right)^{\bar{\Upsilon}}\right) c^{\prime}\left(\operatorname{Ker}\left(\pi_{\infty, 0}^{\mathrm{B}}\right)\right) c^{\prime}\left(\operatorname{Cok}\left(\pi_{\infty, 0}^{\mathrm{A}}\right)\right)=c^{\prime}\left(\operatorname{Ker}\left(\pi_{\infty, 0}^{\mathcal{U} / \mathcal{E}}\right)\right) c^{\prime}\left(\operatorname{Ker}\left(\pi_{\infty, 0}^{\mathrm{A}}\right)\right)
$$

By Theorem 2.4 (iii) and (iv), we have the exact sequence

$$
0 \longrightarrow \mathcal{Q} \otimes \boldsymbol{z}_{p} \operatorname{Ker}\left(\pi_{\infty, 0}^{\mathrm{B}}\right) \longrightarrow\left(\mathcal{Q} \otimes_{\mathbf{z}_{p}} \Lambda_{\Upsilon^{\prime}}\right) / \mathcal{J}_{\bar{D}} \cdot\left(\mathcal{Q} \otimes_{\boldsymbol{z}_{p}} \Lambda_{\Upsilon^{\prime}}\right) \longrightarrow \mathcal{Q} \longrightarrow 0 .
$$

We have seen in the proof of Lemma 7.1 that $\mathcal{J}_{\infty} \cdot\left(\mathcal{Q} \otimes \boldsymbol{Z}_{p} \Lambda_{\Upsilon^{\prime}}\right)$ and $\mathcal{J}_{\bar{D}} \cdot\left(\mathcal{Q} \otimes \boldsymbol{Z}_{p} \Lambda_{\Upsilon^{\prime}}\right)$ are principal ideals. Hence (7.2) implies that

$$
\mathcal{J}_{\infty} c^{\prime}\left(\operatorname{Ker}\left(\pi_{\infty, 0}^{\mathrm{B}}\right)\right)=\mathcal{J}_{\bar{D}} \cdot\left(\mathcal{Q} \otimes_{\mathbf{Z}_{p}} \Lambda_{\Upsilon^{\prime}}\right)
$$

Combining (7.3) with (7.1), we obtain

$$
\mathcal{J}_{\bar{D}} c^{\prime}\left(\left(\mathrm{A}_{\infty}\right)^{\bar{\Upsilon}}\right) c^{\prime}\left(\operatorname{Cok}\left(\pi_{\infty, 0}^{\mathrm{A}}\right)\right)=\mathcal{J}_{\infty} c^{\prime}\left(\operatorname{Ker}\left(\pi_{\infty, 0}^{\mathcal{U} / \mathcal{E}}\right)\right) c^{\prime}\left(\operatorname{Ker}\left(\pi_{\infty, 0}^{\mathrm{A}}\right)\right) .
$$

By (3.1), we have $c^{\prime}\left(\operatorname{Ker}\left(\pi_{\infty, 0}^{\mathcal{U} / \mathcal{E}}\right)\right)=c^{\prime}\left(\operatorname{Cok}\left(\pi_{\infty, 0}^{\mathcal{E}}\right)\right)$. Moreover, by [11, Lemme 4 (2)], we have $c^{\prime}\left(\left(\mathrm{A}_{\infty}\right)^{\bar{\Upsilon}}\right) c\left(\mathrm{~A}_{\infty}\right)=c^{\prime}\left(\left(\mathrm{A}_{\infty}\right) \bar{\Upsilon}\right)$. Considering these two facts, (7.4) becomes

$$
\mathcal{J}_{\bar{D}} c^{\prime}\left(\left(\mathrm{A}_{\infty}\right) \bar{\Upsilon}\right) c^{\prime}\left(\operatorname{Cok}\left(\pi_{\infty, 0}^{\mathrm{A}}\right)\right)=\mathcal{J}_{\infty} c^{\prime}\left(\operatorname{Cok}\left(\pi_{\infty, 0}^{\mathcal{E}}\right)\right) c^{\prime}\left(\operatorname{Ker}\left(\pi_{\infty, 0}^{\mathrm{A}}\right)\right) c\left(\mathrm{~A}_{\infty}\right) .
$$

Combining the obvious relation $c^{\prime}\left(\operatorname{Ker}\left(\pi_{\infty, 0}^{\mathrm{A}}\right)\right) c^{\prime}\left(\mathrm{A}_{\infty, 0}\right)=c^{\prime}\left(\left(\mathrm{A}_{\infty}\right) \bar{\gamma}\right) c^{\prime}\left(\operatorname{Cok}\left(\pi_{\infty, 0}^{\mathrm{A}}\right)\right)$ with (7.5), we derive the lemma. 
Proposition 7.3. We have

$$
\begin{aligned}
& \operatorname{char}_{\mathcal{Q} \otimes \mathbf{z}_{p} \Lambda_{\Gamma}}\left(e_{\chi} \mathcal{Q} \otimes \boldsymbol{Z}_{p} \mathrm{~A}_{\infty}\right) \operatorname{char}_{\mathcal{Q} \otimes \mathbf{z}_{p} \Lambda_{\gamma^{\prime}}}\left(e_{\chi} \mathcal{Q} \otimes \boldsymbol{z}_{p}\left(\mathcal{E}_{\infty, 0} / \mathcal{C}_{\infty, 0}\right)\right) \\
& =\operatorname{char}_{\mathcal{Q} \otimes \mathbf{z}_{p} \Lambda_{\Gamma}}\left(e_{\chi} \mathcal{Q} \otimes \boldsymbol{z}_{p}\left(\mathcal{E}_{\infty} / \mathcal{C}_{\infty}\right)\right) \operatorname{char}_{\mathcal{Q} \otimes \mathbf{z}_{p} \Lambda_{\Upsilon^{\prime}}}\left(e_{\chi} \mathcal{Q} \otimes \mathbf{z}_{p} \mathrm{~A}_{\infty, 0}\right),
\end{aligned}
$$

in $\mathcal{Q} \otimes z_{p} \Lambda_{\Upsilon^{\prime}}$.

Proof. Since $\left(\mathcal{E}_{\infty}\right)^{\bar{\Upsilon}} \subseteq\left(\mathcal{U}_{\infty}\right)^{\bar{\Upsilon}}$, we deduce from Theorem 2.1 that $\left(\mathcal{E}_{\infty}\right)^{\bar{\Upsilon}}=0$. Hence we have the diagram

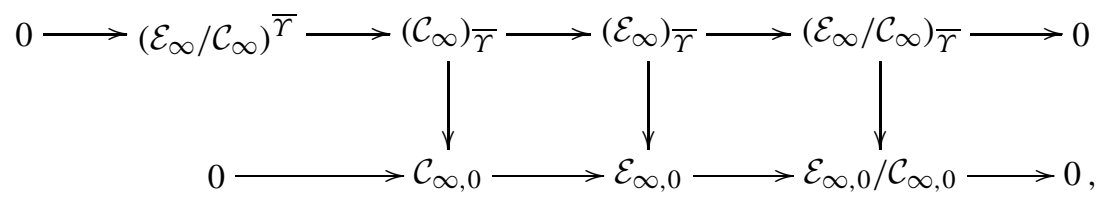

where the rows are exact. We keep the notation of the proof of Lemma 7.2. Since $\operatorname{Ker}\left(\pi_{\infty, 0}^{\mathcal{E}}\right)=$ 0 by (3.1) and $c^{\prime}\left(\operatorname{Ker}\left(\pi_{\infty, 0}^{\mathcal{C}}\right)\right)=\mathcal{Q} \otimes \mathbf{Z}_{p} \Lambda_{\Upsilon^{\prime}}$ by Proposition 4.7, the snake lemma applied to (7.7) gives

(7.8) $\quad c^{\prime}\left(\left(\mathcal{E}_{\infty} / \mathcal{C}_{\infty}\right)^{\bar{\Upsilon}}\right) c^{\prime}\left(\operatorname{Cok}\left(\pi_{\infty, 0}^{\mathcal{C}}\right)\right) c^{\prime}\left(\operatorname{Cok}\left(\pi_{\infty, 0}^{\mathcal{E} / \mathcal{C}}\right)\right)=c^{\prime}\left(\operatorname{Ker}\left(\pi_{\infty, 0}^{\mathcal{E} / \mathcal{C}}\right)\right) c^{\prime}\left(\operatorname{Cok}\left(\pi_{\infty, 0}^{\mathcal{E}}\right)\right)$.

Applying Lemma 7.2 and then Lemma 7.1 to (7.8), we deduce

$$
c\left(\mathrm{~A}_{\infty}\right) c^{\prime}\left(\left(\mathcal{E}_{\infty} / \mathcal{C}_{\infty}\right)^{\bar{\Upsilon}}\right) c^{\prime}\left(\operatorname{Cok}\left(\pi_{\infty, 0}^{\mathcal{E} / \mathcal{C}}\right)\right)=c^{\prime}\left(\mathrm{A}_{\infty, 0}\right) c^{\prime}\left(\operatorname{Ker}\left(\pi_{\infty, 0}^{\mathcal{E} / \mathcal{C}}\right)\right) .
$$

Since $c^{\prime}\left(\operatorname{Ker}\left(\pi_{\infty, 0}^{\mathcal{E} / \mathcal{C}}\right)\right) c^{\prime}\left(\mathcal{E}_{\infty, 0} / \mathcal{C}_{\infty, 0}\right)=c^{\prime}\left(\operatorname{Cok}\left(\pi_{\infty, 0}^{\mathcal{E} / \mathcal{C}}\right)\right) c^{\prime}\left(\left(\mathcal{E}_{\infty} / \mathcal{C}_{\infty}\right) \bar{\gamma}\right),(7.9)$ gives

$$
c^{\prime}\left(\mathcal{E}_{\infty, 0} / \mathcal{C}_{\infty, 0}\right) c\left(\mathrm{~A}_{\infty}\right) c^{\prime}\left(\left(\mathcal{E}_{\infty} / \mathcal{C}_{\infty}\right)^{\bar{\Upsilon}}\right)=c^{\prime}\left(\left(\mathcal{E}_{\infty} / \mathcal{C}_{\infty}\right) \bar{\gamma}\right) c^{\prime}\left(\mathrm{A}_{\infty, 0}\right) .
$$

We deduce the proposition by applying [11, Lemme 4 (2)] in (7.10).

LEMMA 7.4. The image of $\operatorname{char}_{\Lambda_{\infty}}\left(\mathcal{E}_{\infty} / \mathcal{C}_{\infty}\right)$ in $\Lambda_{\Upsilon^{\prime}}$ is prime to $p$.

PROOF. We apply the snake lemma to the diagram

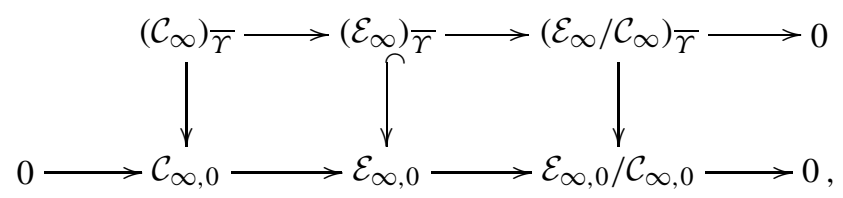

where the second vertical arrow is injective by Lemma 3.1. Then $\operatorname{char}_{\Lambda_{\Upsilon^{\prime}}}\left(\operatorname{Ker}\left(\pi_{\infty, 0}^{\mathcal{E} / \mathcal{C}}\right)\right)$ divides $\operatorname{char}_{\Lambda_{\Upsilon^{\prime}}}\left(\operatorname{Cok}\left(\pi_{\infty, 0}^{\mathcal{C}}\right)\right)$. By Proposition 4.4, we deduce that

$$
\operatorname{char}_{\Lambda_{\gamma^{\prime}}}\left(\operatorname{Ker}\left(\pi_{\infty, 0}^{\mathcal{E} / \mathcal{C}}\right)\right) \text { is prime to } p .
$$

By [20, Theorem 1.1] and Gillard result [3, 3.4. Théorème],

$$
\operatorname{char}_{\Lambda_{\gamma^{\prime}}}\left(\mathcal{E}_{\infty, 0} / \mathcal{C}_{\infty, 0}\right)=\operatorname{char}_{\Lambda_{\Upsilon^{\prime}}}\left(\mathrm{A}_{\infty, 0}\right) \text { is prime to } p .
$$

From (7.12) and (7.13) it follows that $\operatorname{char}_{\Lambda_{\Upsilon^{\prime}}}\left(\mathcal{E}_{\infty} / \mathcal{C}_{\infty}\right) \bar{\Upsilon}$ is prime to $p$. Then Lemma 7.4 follows from Lemma [11, Lemme 4 (2)]. 
Now we prove Theorem 1.1. By Proposition 6.1, there is $g \in \Lambda_{\infty, \chi}$ such that

$$
g \operatorname{char}_{\Lambda_{\infty, \chi}}\left(\mathrm{A}_{\infty, \chi}\right)=\operatorname{char}_{\Lambda_{\infty, \chi}}\left(\mathcal{E}_{\infty} / \mathcal{C}_{\infty}\right)_{\chi} .
$$

By Proposition 2.8, the image of $\operatorname{char}_{\Lambda_{\infty, \chi}}\left(\mathrm{A}_{\infty, \chi}\right)$ in $\boldsymbol{Z}_{p}[\chi]\left[\left[\Upsilon^{\prime}\right]\right]$ is prime to $p$. By (7.13), $\operatorname{char}_{\boldsymbol{Z}_{p}[\chi]\left[\left[\Upsilon^{\prime}\right]\right]}\left(\mathcal{E}_{\infty, 0} / \mathcal{C}_{\infty, 0}\right)_{\chi}$ and $\operatorname{char}_{\boldsymbol{Z}_{p}[\chi]\left[\left[\Upsilon^{\prime}\right]\right]}\left(\mathrm{A}_{\infty, 0, \chi}\right)$ are prime to $p$. From Lemma 7.4 we deduce that the image of $\operatorname{char}_{\Lambda_{\infty, \chi}}\left(\mathcal{E}_{\infty} / \mathcal{C}_{\infty}\right)_{\chi}$ in $\boldsymbol{Z}_{p}[\chi]\left[\left[\Upsilon^{\prime}\right]\right]$ is prime to $p$. Since all the ideals involved are prime to $p$, we deduce from (7.6) that

$$
\begin{aligned}
& \operatorname{char}_{\Lambda_{\infty, \chi}}\left(\mathrm{A}_{\infty, \chi}\right) \operatorname{char}_{Z_{p}[\chi]\left[\left[\Upsilon^{\prime}\right]\right]}\left(\mathcal{E}_{\infty, 0} / \mathcal{C}_{\infty, 0}\right)_{\chi} \\
& \quad=\operatorname{char}_{\Lambda_{\infty, \chi}}\left(\mathcal{E}_{\infty} / \mathcal{C}_{\infty}\right)_{\chi} \operatorname{char}_{Z_{p}[\chi]\left[\left[\gamma^{\prime}\right]\right]}\left(\mathrm{A}_{\infty, 0, \chi}\right)
\end{aligned}
$$

in $Z_{p}[\chi]\left[\left[\Upsilon^{\prime}\right]\right]$. By [1, Theorem 3.1] or [19, Theorem 1.1] for the general case, we know that

$$
\operatorname{char}_{Z_{p}[\chi]\left[\left[\Upsilon^{\prime}\right]\right]}\left(\mathcal{E}_{\infty, 0} / \mathcal{C}_{\infty, 0}\right)_{\chi}=\operatorname{char}_{Z_{p}[\chi]\left[\left[\Upsilon^{\prime}\right]\right]}\left(\mathrm{A}_{\infty, 0, \chi}\right) \text {. }
$$

Combining (7.16) and (7.14) with (7.15), we obtain that the image of $g$ in $Z_{p}[\chi]\left[\left[\Upsilon^{\prime}\right]\right]$ is a unit. Then $g$ must be a unit of $\Lambda_{\infty, \chi}$, and Theorem 1.1 follows.

Acknowledgments. I would like to thank the referee for his valuable comments.

\section{REFERENCES}

[1] W. BLEY, Equivariant Tamagawa number conjecture for abelian extensions of a quadratic imaginary field, Doc. Math. 11 (2006), 73-118.

[ 2 ] E. DE Shalit, Iwasawa theory of elliptic curves with complex multiplication. $p$-adic $L$ functions, Perspect. Math. 3, Academic Press, Inc., Boston, MA, 1987.

[ 3 ] R. Gillard, Fonctions $L$-adiques des corps quadratiques imaginaires et de leurs extensions abéliennes, J. Reine Angew. Math. 358 (1985), 76-91.

[ 4 ] R. GreEnBerg, On the structure of certain Galois groups, Invent. Math. 47 (1978), 85-99.

[ 5 ] C. GReither, Class groups of abelian fields, and the main conjecture, Ann. Inst. Fourier (Grenoble) 42 (1992), 445-499.

[6] K. IWASAWA, On $\boldsymbol{Z}_{l}$-extensions of algebraic number fields, Ann. of Math. 98 (1973), 246-326.

[ 7 ] A. Jilali And H. Oukhaba, Stark units in $\boldsymbol{Z}_{p}$-extensions, Funct. Approx. Comment. Math. 45 (2011), part I, 105-124.

[ 8 ] J. JOHNSON-LEUNG AND G. KingS, On the equivariant main conjecture for imaginary quadratic fields, J. Reine Angew. Math. 653 (2011), 75-114.

[ 9 ] H. OukнавA, On Iwasawa theory of elliptic units and 2-ideal class groups, J. Ramanujan Math. Soc. 27 (2012), no. 3, 255-373.

[10] H. Oukhaba and S. Viguié, The Gras conjecture in function fields by Euler systems, Bull. Lond. Math. Soc. 43 (2011), 523-535.

[11] B. PERrin-Riou, Arithmétique des courbes elliptiques et théorie d'Iwasawa, Supplément au Bulletin de la société mathématique de France 112 n 4 Mém. Soc. Math. France (N.S.) No. 17 (1984), 130 pp.

[12] G. Robert, Unités elliptiques, Bull. Soc. Math. France No 36, Bull. Soc. Math. France, Tome 101, Société Mathématique de France, Paris, 1973.

[13] G. Robert, Unités de Stark comme unités elliptiques, Prépublication de l'Institut Fourier 143, 1989.

[14] G. ROBERT, Concernant la relation de distribution satisfaite par la fonction $\varphi$ associée à un réseau complexe, Invent. Math. 100 (1990), 231-257.

[15] K. RUBIN, On the main conjecture of Iwasawa theory for imaginary quadratic fields, Invent. Math. 93 (1988), 701-713. 
[16] K. RUBIN, The "main conjectures" of Iwasawa theory for imaginary quadratic fields, Invent. Math. 103 (1991), 25-68.

[17] K. Rubin, More "main conjectures" for imaginary quadratic fields. Elliptic curves and related topics, 23-28, CRM Proc. Lecture Notes 4, Amer. Math. Soc., Providence, RI, 1994.

[18] J. TATE, Les conjectures de Stark sur les fonctions L d'Artin en $s=0$, Lecture notes edited by Dominique Bernardi and Norbert Schappacher, Progr. Math. 47, Birkhäuser Boston, Inc., Boston, MA, 1984.

[19] S. VIGUIÉ, On the classical main conjecture for imaginary quadratic fields, to appear in JP J. Algebra Number Theory Appl.

[20] S. VIGUiÉ, Global units modulo elliptic units and ideal class groups, Int. J. Number Theory 8 (2012), 569-588.

[21] J.-P. Wintenberger, Structure galoisienne de limites projectives d'unités locales, Compositio Math. 42 (1980/81), 89-103.

MATHEMATISChES INSTITUT

DER UNIVERSITAT MUNCHEN

THERESIENSTRASSE 39

BURO 413 D-80333

MUNCHEN

GERMANY

E-mail address: stephane.viguie@math.lmu.de 